\title{
Anakinra restores cellular proteostasis by coupling mitochondrial redox balance to autophagy
}

\author{
Frank L. van de Veerdonk, ${ }^{1}$ Giorgia Renga, ${ }^{2}$ Marilena Pariano, ${ }^{2}$ Marina M. Bellet, ${ }^{2}$ Giuseppe Servillo, ${ }^{2}$ Francesca Fallarino, ${ }^{2}$ \\ Antonella De Luca, ${ }^{2}$ Rossana G. Iannitti, ${ }^{2}$ Danilo Piobbico, ${ }^{2}$ Marco Gargaro, ${ }^{2}$ Giorgia Manni, ${ }^{2}$ Fiorella D'Onofrio, ${ }^{2}$ \\ Claudia Stincardini, ${ }^{2}$ Luigi Sforna, ${ }^{2}$ Monica Borghi, ${ }^{2}$ Marilena Castelli, ${ }^{2}$ Stefania Pieroni, ${ }^{2}$ Vasileios Oikonomou, ${ }^{2}$ Valeria R. Villella, ${ }^{3}$ \\ Matteo Puccetti, ${ }^{4}$ Stefano Giovagnoli, ${ }^{4}$ Roberta Galarini, ${ }^{5}$ Carolina Barola, ${ }^{5}$ Luigi Maiuri, ${ }^{3,6}$ Maria Agnese Della Fazia, ${ }^{2}$ \\ Barbara Cellini, ${ }^{2}$ Vincenzo Nicola Talesa, ${ }^{2}$ Charles A. Dinarello, ${ }^{1,7}$ Claudio Costantini, ${ }^{2}$ and Luigina Romani ${ }^{2}$ \\ 'Department of Medicine, Radboud University Medical Center, Nijmegen, Netherlands. ${ }^{2}$ Department of Medicine and Surgery, University of Perugia, Perugia, Italy. ${ }^{3}$ European Institute for Research in Cystic \\ Fibrosis, Division of Genetics and Cell Biology, San Raffaele Scientific Institute, Milan, Italy. ${ }^{4}$ Department of Pharmaceutical Sciences, University of Perugia, Perugia, Italy. ${ }^{5}$ sstituto Zooprofilattico Sperimentale \\ dell'Umbria e delle Marche "Togo Rosati," Perugia, Italy. Department of Health Sciences, University of Piemonte Orientale, Novara, Italy. ${ }^{~}$ Department of Medicine, University of Colorado, Aurora, Colorado, USA.
}

\begin{abstract}
Autophagy selectively degrades aggregation-prone misfolded proteins caused by defective cellular proteostasis. However, the complexity of autophagy may prevent the full appreciation of how its modulation could be used as a therapeutic strategy in disease management. Here, we define a molecular pathway through which recombinant IL-1 receptor antagonist (IL1Ra, anakinra) affects cellular proteostasis independently from the IL-1 receptor (IL-1R1). Anakinra promoted $\mathrm{H}_{2} \mathrm{O}_{2}-\mathrm{driven}$ autophagy through a xenobiotic sensing pathway involving the aryl hydrocarbon receptor that, activated through the indoleamine 2,3-dioxygenase 1-kynurenine pathway, transcriptionally activated NADPH oxidase 4 independent of the IL-1R1. By coupling the mitochondrial redox balance to autophagy, anakinra improved the dysregulated proteostasis network in murine and human cystic fibrosis. We anticipate that anakinra may represent a therapeutic option in addition to its IL-1R1dependent antiinflammatory properties by acting at the intersection of mitochondrial oxidative stress and autophagy with the capacity to restore conditions in which defective proteostasis leads to human disease.
\end{abstract}

\section{Introduction}

The lung is equipped with a robust proteostasis network for handling protein folding, misfolding, unfolding, and degradation in response to mechanical and environmental stress (1). Consistent with the emerging importance of the autophagy pathway in multiple human pulmonary diseases (2), autophagy is required in the maintenance of cellular proteostasis (3). Autophagy selectively degrades aggregation-prone misfolded proteins, such as those involved in the pathogenesis of certain neurodegenerative and lung diseases and aging (4). In certain types of lung diseases, autophagy may have a protective role to allow handling of misfolded proteins and prevent inflammatory damage. In cystic fibrosis (CF), an autosomal recessive disorder caused by mutations in the gene encoding for the $\mathrm{CF}$ transmembrane conductance regulator (CFTR; ref. 5), disruption of autophagy could promote pathogenesis of the disease (6). Defective autophagy has indeed been observed in cells expressing the most common mutant variant p.Phe508del-CFTR and accumulating polyubiquitinated proteins and aggresome-like structures. Given that functional autophagy

Authorship note: LM is deceased.

Conflict of interest: Conflict of interest statement: The authors have declared that no conflict of interest exists.

Copyright: @ 2022, van de Veerdonk et al. This is an open access article published under the terms of the Creative Commons Attribution 4.0 International License.

Submitted: October 7, 2020; Accepted: November 24, 2021; Published: January 18, 2022. Reference information: J Clin Invest. 2022;132(2):e144983.

https://doi.org/10.1172/JCl144983. and proteostasis have been shown to promote trafficking, plasma membrane targeting, and stability of defective CFTR $(7,8)$, strategies aiming at restoring autophagy have been proposed as a therapeutic approach in $\mathrm{CF}(7,9)$. Cysteamine and an endogenously occurring nitric oxide donor are well-known examples of proteostasis and autophagy regulators endowed with therapeutic efficacy in preclinical CF (6). However, the complexity of autophagy may prevent the full appreciation of how its modulation could be used as a therapeutic strategy in disease management. This complexity necessitates preclinical studies aimed at defining the molecular pathways of autophagy and their relevance to disease pathogenesis.

Over the past 30 years, IL-1-mediated inflammation has been established in a broad spectrum of diseases. Quenching IL-1-mediated inflammation with anakinra has provided clinically evident benefits associated with an unparalleled record of safety. By mimicking the naturally occurring IL-1 receptor antagonist (IL-1Ra), anakinra - which differs from IL-1Ra by the presence of an extra methionine in the amino terminus and the absence of glycosylation - exerts its effects by inhibiting the binding of IL- $1 \beta$ and IL- $1 \alpha$ to IL-1 receptor (IL-1R1) (10). Anakinra is presently in clinical trials to treat cancer, and off-label use of anakinra far exceeds its approved indications $(10,11)$. This raises the intriguing hypothesis that anakinra may have effects beyond its receptor-inhibiting activity. Besides a secreted protein, 3 intracellular, un-secreted isoforms of IL-1Ra have been described in humans and in mouse tissues (12). Whereas extracellular IL-1Ra inhibits IL-1 activity by binding to IL-1R1, intracellular IL-1Ra1 was shown to exhibit antiinflammatory properties (13), thus 

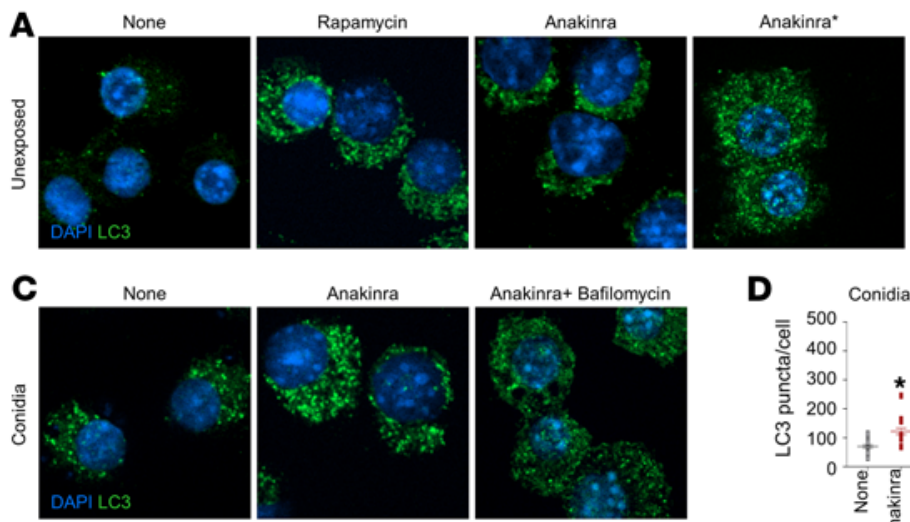

E

None

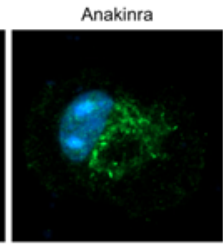

Anakinra+ Bafilomycin
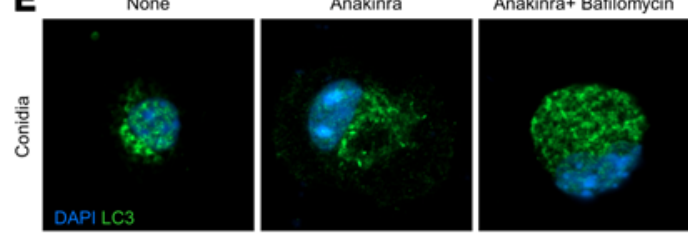

D
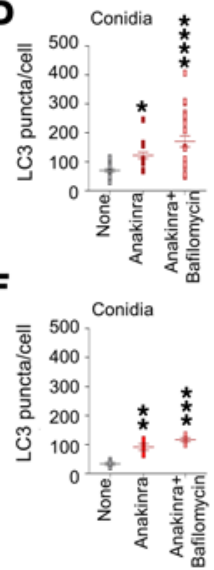

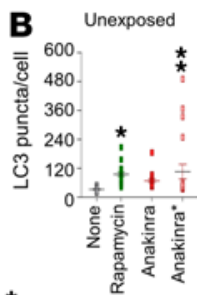

G
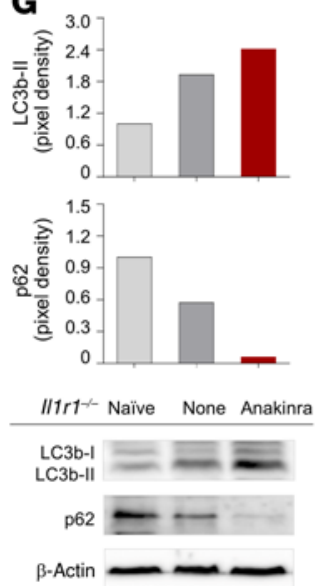

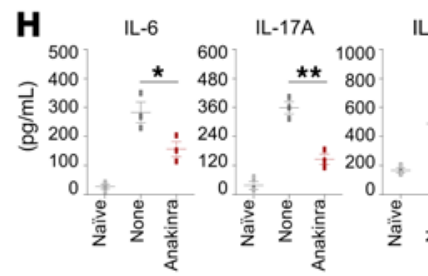

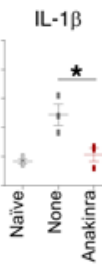
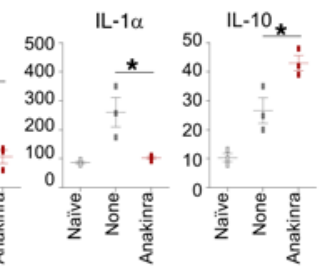

Figure 1. Anakinra induces autophagy and limits inflammation in the absence of IL-1R1. (A-D) LC3 staining of RAW 264.7 cells treated with 50 $\mu \mathrm{M}$ rapamycin, $10 \mu \mathrm{g} / \mathrm{mL}$ anakinra, or a truncated form (Anakinra*) (A and B); exposed to A. fumigatus conidia and treated with anakinra with and without $100 \mathrm{nM}$ bafilomycin for 4 hours (C and $\mathbf{D}$ ). ( $E$ and $\mathbf{F}$ ) LC3 staining of alveolar macrophages purified from $I / 171^{-/-}$naive mice exposed to $A$. fumigatus conidia and treated with anakinra and/or bafilomycin as above. (B, D, and F) Mean percentage of LC3 puncta/cells $(n=4-22)$. Data represent the mean \pm SEM of 1 representative out of $3(\mathbf{A}-\mathbf{D})$ or 2 (E and $\mathbf{F}$ ) independent experiments. DAPI was used to detect nuclei. (G-I) $\| 11 r^{-1-}$ mice were infected (i.n.) with live $A$. fumigatus conidia, treated with $10 \mathrm{mg} / \mathrm{kg}$ anakinra (i.p.) as described in Methods, and assessed for immunoblotting of LC3b and p62 (G), cytokine production (ELISA) in lung homogenates $(\mathbf{H})$, and lung histology [periodic acid-Schiff (PAS) staining] (I) at 7 days after infection. Scale bar: $200 \mu \mathrm{m}$. (G and I) A representative experiment is shown; (H) data represent 3 independent experiments. Each independent in vivo experiment includes 6-8 mice per group pooled before analysis. ${ }^{*} P<0.05,{ }^{* *} P<0.01,{ }^{* *} P<0.001,{ }^{* * * *} P$ $<0.0001$, treated versus untreated (None) cells. One-way ANOVA, Bonferroni post hoc test. suggesting that anakinra may have a receptor-independent activity. In this regard, the facts that (a) anakinra has shown a superior activity compared with IL-1 $\beta$ neutralizing antibody on murine lung infection and pathology (14), (b) cellular uptake of exogenous anakinra has been described (15), and (c) surface-bound IL-1Ra does not undergo receptor-mediated internalization (16) all suggest that anakinra may exert receptor-independent intracellular activities.

In the present study, we have characterized the process of autophagy induced by anakinra, its dependency on receptor activity, and its possible role in lung proteostasis. By using preclinical models of CF in vitro and in vivo, we have discovered that anakinra promotes IL-1R1-independent autophagy through the aryl hydrocarbon receptor that, activated through the indoleamine 2,3-dioxygenase 1-kynurenine pathway, leads to NOX4-dependent mitochondrial $\mathrm{H}_{2} \mathrm{O}_{2}$ production and positively regulates cellular proteostasis in mice and human cells with the p.Phe508del-CFTR mutation.

\section{Results}

Anakinra induces IL-1R1-independent autophagy. We have already shown that anakinra dose-dependently decreased inflammasome activity in Aspergillus fumigatus infection via autophagy $(14,17)$.
We found here that anakinra promoted autophagy in the relative absence of IL-1R1 both in vitro and in vivo. In RAW 264.7 cells in which defective NLRP3 activity (18) allows uncoupling of the intracellular effects of anakinra from the inflammasome (Figure 1, A-D) and in purified $\mathrm{Illr}^{-/-}$lung macrophages (Figure 1, E and F), anakinra increased the number of LC3 punctae in cells either unexposed or after exposure to Aspergillus conidia. LC3 expression was increased in the presence of either bafilomycin (Figure 1, C and D) or chloroquine (Supplemental Figure 1; supplemental material available online with this article; https://doi.org/10.1172/JCI144983DS1), suggesting that anakinra induces an increase in autophagosomal turnover. Autophagy was still observed with a truncated form of anakinra obtained by limited proteolysis of the N-terminus, likely affecting the receptor binding site of the molecule (ref. 19, Figure 1, $A$ and B, and Supplemental Figure 2). In vivo, treatment of Aspergillus-infected Illr1 $1^{-/-}$mice with anakinra increased the ratio of LC3II/I and decreased p62, a ubiquitin-binding protein that is selectively degraded by autophagy (Figure 1G); inhibited IL-6, IL-17A, IL-1 $\beta$, and IL-1 $\alpha$ production; increased IL-10 (Figure $1 \mathrm{H}$ ); and ameliorated lung inflammation (Figure 1I). Similar results were obtained in Pseudomonas aeruginosa-infected $I l 1 \mathrm{r}^{-/-}$mice (Supplemental Figure 
A

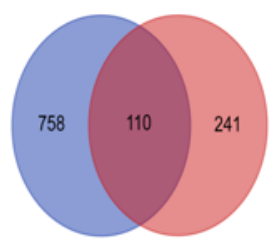

B

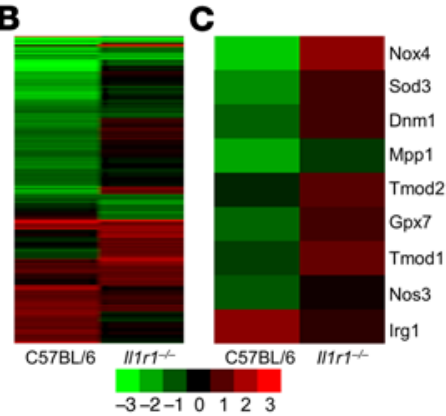

D

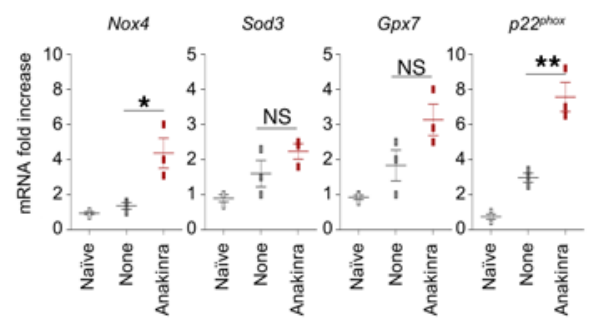

E

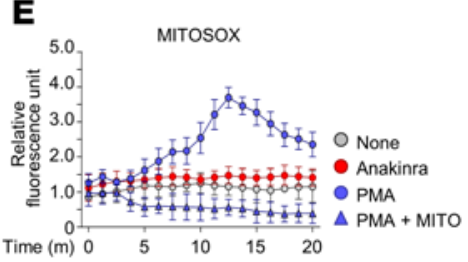

$\mathbf{F}$

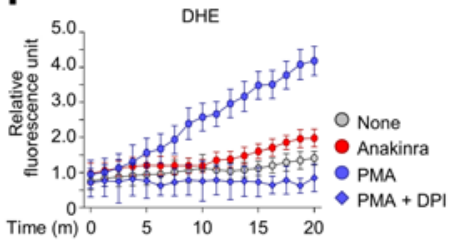

G

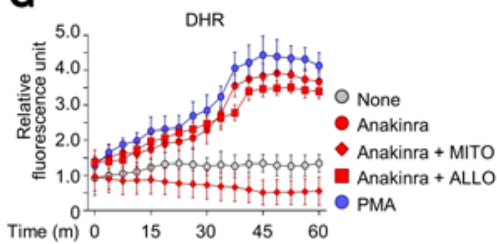

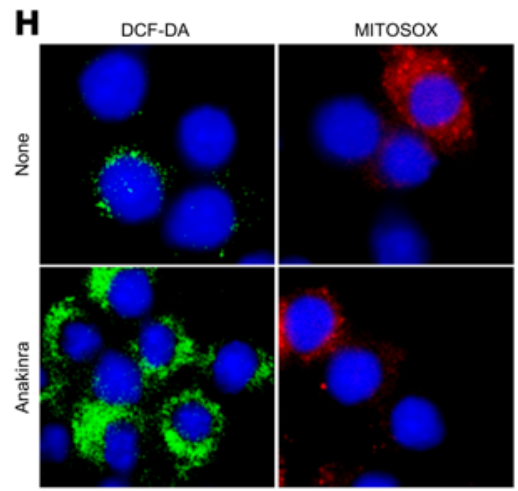
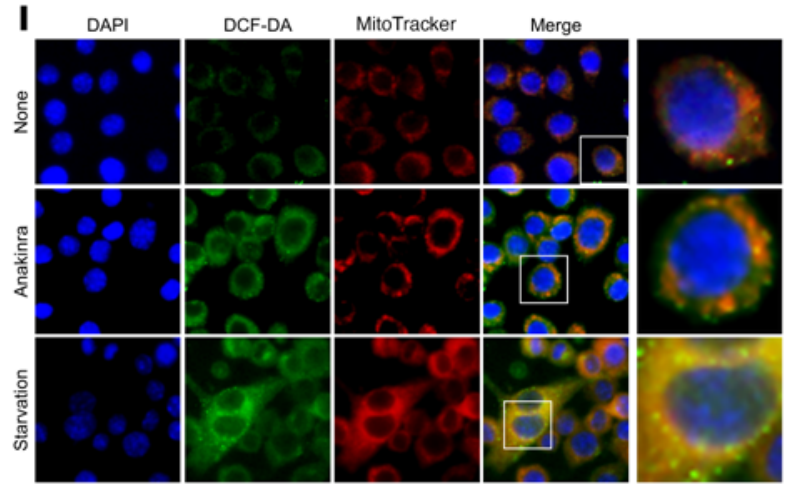

Figure 2. Anakinra affects mitochondrial redox balance in the absence of IL-1R1. (A) Genes differentially expressed in response to anakinra in purified alveolar macrophages from C57BL/6 and $117 r^{-1-}$ mice stimulated in vitro with $10 \mu \mathrm{g} / \mathrm{mL}$ of anakinra for 4 hours. (B and C) Hierarchical clustering (fold change $>2, P$ value $<0.05$, FDR $<0.05$ ) by RNA-Seq of all genes (B) and genes involved in oxidative stress (C). (D) RT-PCR on total lung cells from $\| 11 r^{-/-}$mice at 7 days after $A$. fumigatus i.n. infection. ${ }^{*} P<0.05$ and ${ }^{* *} P<0.01$, anakinra-treated versus untreated (None) mice. NS, not statistically significant. One-way ANOVA, Bonferroni post hoc test. Data are the mean \pm SEM of 1 representative out of 3 independent experiments. (E-C) RAW 264.7 cells were exposed to $10 \mathrm{ng} / \mathrm{mL}$ PMA or $10 \mu \mathrm{g} / \mathrm{mL}$ anakinra for 4 hours at $37^{\circ} \mathrm{C}$ in the presence of diphenylene iodonium (DPI), allopurinol (ALLO), or MitoTEMPO (MITO) with the addition of MitoSOX red (E), DHE (F), and DHR (G) for mitochondrial superoxide, NADPH-dependent superoxide, and mitochondrial hydrogen peroxide assessment, respectively. Fluorescence was measured at 510 excitation and 580 emission. The results presented for all fluorimetric measurements are the mean \pm SEM of 1 representative out of 3 independent experiments, tested in duplicate. Two-way ANOVA, Bonferroni post hoc test. (H) Fluorescence of RAW 264.7 cells exposed to anakinra and stained with DCF-DA or MitoSOX red. (I) RAW 264.7 cells were starved or exposed to anakinra before visualization (magnified in the last column) of $\mathrm{H}_{2} \mathrm{O}_{2}$ /mitochondria colocalization by staining with DCF-DA and MitoTracker red probes, respectively. Starvation was carried out in Earle's balanced salt solution. DAPI was used to detect nuclei. Images were acquired using the Olympus BX51 fluorescence microscope. For $P$ value, see Supplemental Figure 5.

3). These findings suggest that anakinra promotes autophagy and shows antiinflammatory activity beyond its competitive inhibition of IL-1 binding to IL-1R1.

Anakinra affects mitochondrial redox balance via NOX4. To understand the molecular mechanisms behind our observation of IL-1R1-independent activity of anakinra, we assessed gene expression in purified C57BL/6 and Illr1 ${ }^{-/}$alveolar macrophages exposed to anakinra in vitro to identify genes that are specifically influenced by anakinra in the absence of IL-1R1. RNA-Seq data analysis revealed that 1109 genes (Figure 2, A and B and Supplemental Table 1) were modulated by anakinra in both types of murine cells. Gene ontology (GO) functional classification analysis of differentially expressed genes revealed that 3 main biological processes were involved, i.e., biological regulation, response to stimulus, and response to stress (Supplemental Figure 4). Accordingly, genes involved in the oxidative stress and antioxidative defense pathways, known to be involved in the activation of autophagy (20), were significantly upregulated in $I l 1 r 1^{-/-}$cells by anakinra, mostly Nox4, followed by Sod 3 and Gpx7, among others (Figure 2C and Supplemental Table 2). In vivo, Nox4, more than Sod3 or Gpx7, gene expression was significantly induced by anakinra in the lungs of infected Illr1-/mice along with the catalytic subunit $p 22^{\text {phox }}$ gene (Figure 2D). NOX4 plays a pivotal role in autophagy (21). Unlike the other NOX isoforms, NOX4 directly produces $\mathrm{H}_{2} \mathrm{O}_{2}$ because of its unique E loop and intrinsic capacity to induce $\mathrm{H}_{2} \mathrm{O}_{2}$ and can be localized in mitochondria $(22,23)$. As a next step, we measured ROS production by anakinra in RAW 264.7 cells. In line with anakinra inducing NOX4 activity, anakinra did not induce mitochondrial (Figure 
2E) or NADPH oxidase-dependent superoxide $\mathrm{O}_{2}^{-}$(Figure $2 \mathrm{~F}$ ) but induced mitochondrial $\mathrm{H}_{2} \mathrm{O}_{2}$, as revealed by (a) dihydrorhodamine 123 (DHR) fluorescence in the presence of allopurinol (that scavenges xanthine-oxidase-dependent ROS) but not MitoTEMPO (that scavenges mitochondrial ROS) (Figure 2G); (b) the positive immunostaining of anakinra-treated cells with 2',7'-dichlorofluorescin diacetate (DCF-DA), a probe that reacts mainly with $\mathrm{H}_{2} \mathrm{O}_{2}$ to form a fluorescent compound but not MitoSOX (that detects mitochondrial $\mathrm{O}_{2}^{-}$) (Figure $2 \mathrm{H}$ ); and (c) the positive double immunostaining with MitoTracker red, a marker for functional mitochondria, and DCF-DA. Similar to what was observed in starvation conditions leading to $\mathrm{H}_{2} \mathrm{O}_{2}$ production that induces autophagy, all the DCF punctae colocalized with MitoTracker-stained structures (Figure 2I and Supplemental Figure 6 for colocalization indexes). These data strongly support mitochondria being the source of the $\mathrm{H}_{2} \mathrm{O}_{2}$ induced by anakinra. Experiments in vitro in purified $\mathrm{Illr}^{-1-}$ alveolar macrophages and in vivo in infected $I l 1 r 1^{-/-}$mice confirmed that $\mathrm{H}_{2} \mathrm{O}_{2}$ production by anakinra was IL-1R1 independent and also occurred in the presence of IL-1R1 (Supplemental Figure 7).

Anakinra promotes autophagy via mitochondrial $\mathrm{H}_{2} \mathrm{O}_{2}$. A local rise in $\mathrm{H}_{2} \mathrm{O}_{2}$ in the vicinity of mitochondria leads to lipidation of Atg8, an essential step in the process of autophagy, via the redox-sensitive Atg 4 cysteine protease (24). Atg 4 enables the conversion of LC3-I to lipidated LC3-II, its insertion into the autophagosome, and the recycling of LC3-II after autophagosome-lysosome fusion. Given that anakinra did not affect the viability of the cells and actually retarded senescence (Supplemental Figure 8), which is induced by autophagy (25), we hypothesized that the $\mathrm{H}_{2} \mathrm{O}_{2}$ induced by anakinra may serve as a signaling molecule for autophagy. We evaluated $\mathrm{H}_{2} \mathrm{O}_{2} / \mathrm{LC} 3$ colocalization in starved cells and in anakinra-treated RAW 264.7 cells exposed to Aspergillus conidia while inhibiting Atg4a by RNA interference. Similar to starved cells, $\mathrm{H}_{2} \mathrm{O}_{2}$ colocalized with LC3-stained phagosome in anakinra-treated cells; blocking of Nox4 or Atg 4 a prevented $\mathrm{H}_{2} \mathrm{O}_{2} / \mathrm{LC} 3$ colocalization induced by anakinra (Figure $3 \mathrm{~A}$ and Supplemental Figure 9), suggesting that as in amino acid starvation (24), anakinra induces autophagy through the NOX4-dependent mitochondrial $\mathrm{H}_{2} \mathrm{O}_{2} /$ Atg 4 signaling pathway. Blocking Nox4 also prevented anakinra-induced autophagy and control of fungal growth and inflammation in vivo in infected $I l 1 r 1^{-/-}$mice (Figure 3, B-E).

Anakinra activates a xenobiotic sensing pathway via IDO1. Since anakinra induces autophagy via mitochondrial $\mathrm{H}_{2} \mathrm{O}_{2}$, independent of the IL-1R1, we explored potential mechanisms of cell entry not linked to IL-1R1. On assessing the uptake of FITC-anakinra in purified alveolar macrophages from C57BL/6 and $I l 1 \mathrm{rl}^{-/-}$mice by immunofluorescence imaging, we were able to detect anakinra intracellularly, particularly in Illr1 $\mathrm{I}^{-/-}$cells (Figure $4 \mathrm{~A}$ and confirmed by Western blot in Figure 4C), where the uptake was inhibited by cytochalasin D (Figure 4B). Therefore, consistent with previous findings (15), anakinra enters the cell independently of the IL-1R1 and might induce autophagy by interacting with intracellular mechanisms of autophagy. A general mechanism to which cells react to xenobiotics is via activation of cellular xenosensors that modulate gene expression that results in the generation of ROS and antioxidant proteins, thereby establishing a regulatory circuit and controlling processes such as apoptosis and autophagy (26). One such xenosensor is the aryl hydrocarbon receptor (AhR), which is known to modulate autophagy and inflammasome activity $(27,28)$. Preliminary experiments revealed that anakinra, in addition to Nox4, upregulated the expression of the AhR-target genes Cyp1a1, Cyp1b1, and Ahrr in $I l 1 r 1^{-/-}$total lung cells in vitro and in lungs of Illr $^{-1 /}$ mice in vivo, albeit to a lesser extent compared with canonical AhR ligands (Supplemental Figure 10). Immunofluorescence staining revealed that anakinra colocalized with AhR in $I l 1 \mathrm{rI}^{-/-}$cells, macrophages (Figure 4D and Supplemental Figure 6), and mouse embryonic fibroblast cells (Figure 4E and Supplemental Figure 6). Colocalization was higher in $I l 1 r 1^{-/-}$than C57BL/6 cells, likely because of the higher AhR protein expression in Illr1 $^{-/-}$mice (Supplemental Figure 11). Despite the colocalization, the failure to show a direct association of anakinra with AhR by IP assay (Figure 4F) prompted us to assess how anakinra would engage AhR and activate its signaling. Consistent with the failure to detect a direct association with $\mathrm{AhR}$, anakinra, as opposed to the AhR ligand kynurenine, did not activate $A h R$ in a standard luciferase reporter assay (Figure $5 \mathrm{~A}$ ), thus confirming that it was not working as a direct AhR ligand. We then assessed whether anakinra may activate AhR via the production of putative intracellular AhR ligands. In this regard, we found that anakinra (a) promoted the ligand-dependent (29) dissociation of AhR from chaperons HSP9O and the AhR-interacting protein AIP (Figure 5B); (b) promoted the complex of AhR with the partner molecule ARNT (AhR nuclear translocator), required for promoter activity (Figure 5C); and (c) activated genes involved in tryptophan (trp) catabolism (Ido1, Ido2, Tdo2, Haao, and Kmo) together with the AhR-dependent genes (Cyp1a1 and Cyp1b1) (Figure 5, D and E). Upon validation, beside the AhR-target genes, Cyp1a1, Cyp1b1, and the AhR repressor Ahrr, the IDO1 gene only was progressively upregulated by anakinra (Figure 5F). Of interest, early after anakinra exposure, SOCS3, which drives proteasomal degradation of IDO1 (30), was downregulated and IDO1 protein expression increased (Figure 5F), a finding suggesting that IDO1 activity may be promoted via transcriptional and posttranscriptional mechanisms. These findings indicate that anakinra may induce trp metabolism along the kynurenine pathway known to generate AhR ligands (31). We measured the levels of kynurenine and downstream metabolites in Il1r1 $1^{--} \mathrm{MEF}$ cells exposed to anakinra and found that kynurenine (Figure 5G) and no other metabolites (Supplemental Table 3) were detected in a manner similar to the IDO1-inducer IFN- $\gamma$, a finding paralleling the activation of Ido1 gene and downregulation of the Kynu gene with kynureninase activity (Figure 5E). Blocking IDO1 prevented both the production of kynurenine and the activation of AhR (Figure $5 \mathrm{H}$ ), indicating that anakinra activates AhR via the IDO1-kynurenine pathway.

Anakinra activates the $\mathrm{A} h \mathrm{R} / \mathrm{Nox} 4 / \mathrm{H}_{2} \mathrm{O}_{2}$ cellular pathway in vivo. The role of AhR was confirmed by further studies showing that anakinra failed to promote autophagy or decrease IL-1 $\beta$, IL-6, and IL-17A production and tissue inflammation in $\mathrm{Ahr}^{-/}$mice (Figure 6, A-C). Moreover, neither Nox4/p22 phox (Figure 6D) nor mitochondrial $\mathrm{H}_{2} \mathrm{O}_{2}$ production (Figure $6 \mathrm{E}$ ) were induced by anakinra in these mice, further proving that induction of NOX4 and mitochondrial $\mathrm{H}_{2} \mathrm{O}_{2}$ by anakinra is dependent on AhR. By using ChIPPCR assay, we were able to demonstrate that AhR binds to the binding site predicted by the ALGGEN-PROMO database and the Eukaryotic Promoter Database of the Nox4 promoter in $\mathrm{Illr}^{-/-}$cells (Figure 6, F and G). Thus, anakinra enters myeloid cells through an 
A

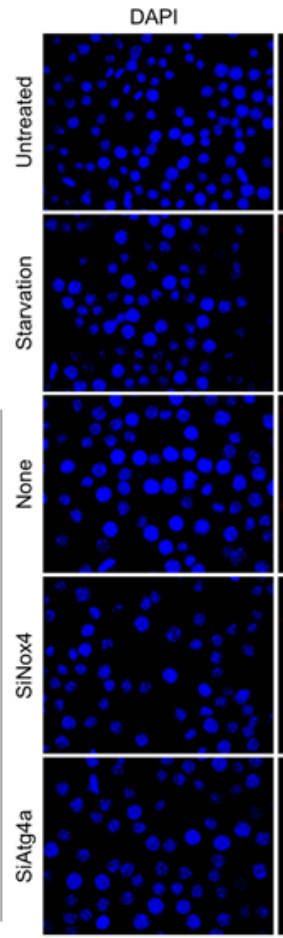

B

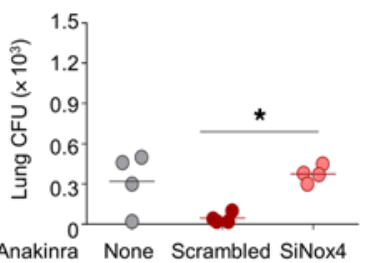

D

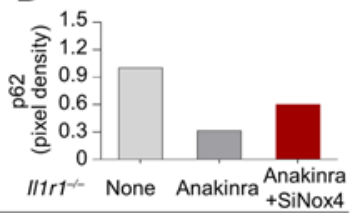

p62

$\beta$-Tubulin
DHR

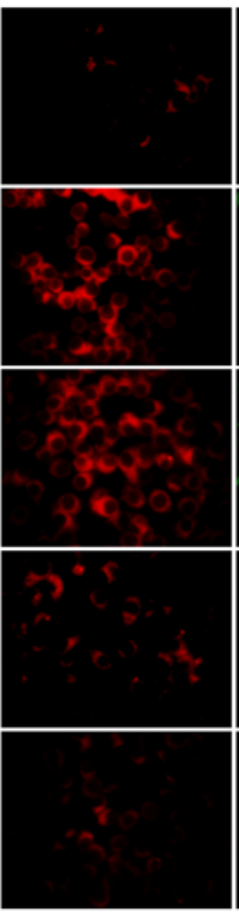

C

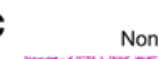

LC3

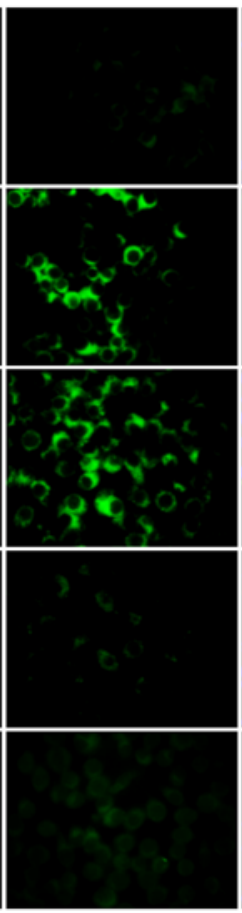

Merge

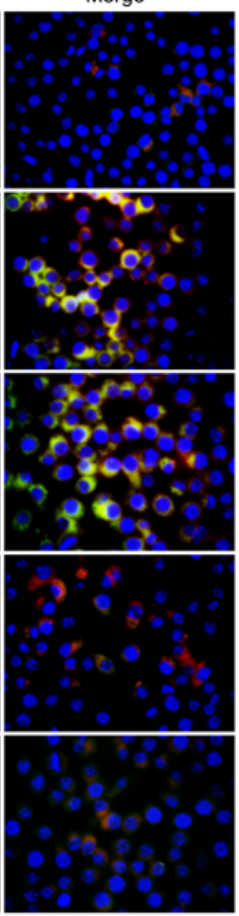

Anakinra

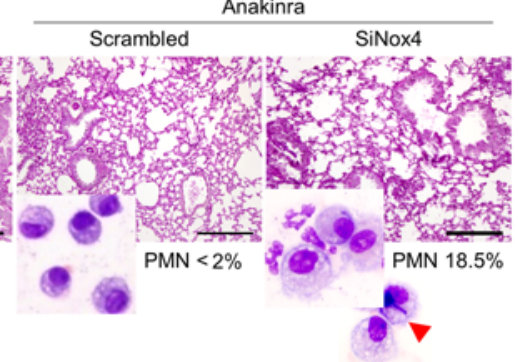

E

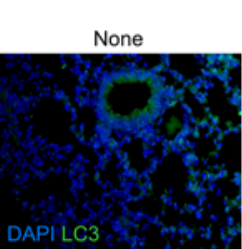

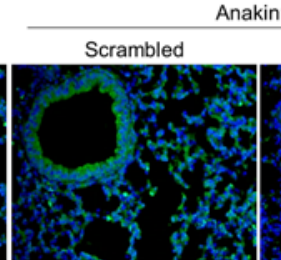

nakinra

SiNox4

Figure 3. Anakinra promotes autophagy via mitochondrial $\mathrm{H}_{2} \mathrm{O}_{2}$. (A) Fluorescence images of EGFP-LC3-transfected RAW264.7 cells exposed to anakinra and A. fumigatus conidia for 4 hours alone or upon inhibition of Atg $4 a$ or Nox4 by SiRNA. None, cells exposed to scrambled RNA. $\mathrm{H}_{2} \mathrm{O}_{2}$ was visualized using $10 \mu \mathrm{M}$ DHR. Images were acquired using the Olympus BX51 fluorescence microscope and analySIS image processing software (Olympus). DAPI was used to detect nuclei. Data are from 1 representative out of 3 independent experiments. (B-E) $\| 17 r^{-/-}$mice were infected and treated with anakinra as in legend to Figure 1, administered SiNox4 or scrambled peptide, and assessed for fungal growth ( $\log _{10}$ CFU) (B), histology [PAS staining] I, p62 immunoblotting (D), and LC3 immunofluorescence staining $(\mathbf{E})$ in lung cell lysates or lungs. Insets in C show the bronchoalveolar lavage morphometry with the percentage of polymorphonuclear cells (PMNs) and an arrow indicating the appearance of fungi in SiNox4-treated mice. For CFU, lung staining, and immunoblotting, data are representative of 1 out of 2 independent experiments. Each independent in vivo experiment includes 4 mice per group. ${ }^{*} P$ $<0.05$, SiNox4-treated versus scrambled peptide. One-way ANOVA, Bonferroni post hoc test. Effectiveness of silencing was verified by quantitative RT-PCR analysis at 24 hours (Supplemental Figure 9). actin-dependent fluid pinocytosis and promotes AhR-dependent Nox4 transcription independent of IL-1R1, which in turn induces autophagy via Atg 4 . An AhR/Nox4 $/ \mathrm{H}_{2} \mathrm{O}_{2}$ cellular pathway interfering with AhR endogenous regulation has already been shown (32).

Anakinra restores functional cellular proteostasis via autophagy in $C F$. To demonstrate the clinical relevance of this newly identified mechanism, we used preclinical models of CF, a prototypical disease of dysregulated proteostasis that is linked to defective autophagy and aberrant inflammasome-mediated inflammation (14). We investigated whether anakinra, in addition to its receptordependent activity in CF (14), would affect trafficking of CFTR via secretory autophagy, a form of nondegradative autophagy that participates in the unconventional secretion of cytoplasmic entities (33) and involves an oxidative stress (34). We used 2 complementary preclinical models involving homozygous F508del-Cftr
C57BL/6 mice (referred to as $C f t r^{F 508 d e l}$ mice) treated with anakinra in vivo and human cells bearing the p.Phe508del-CFTR mutation exposed to anakinra in vitro. We found that anakinra promoted Nox4 gene expression (Figure 7A) and $\mathrm{H}_{2} \mathrm{O}_{2}$ production (Figure 7B) and restored CFTR expression in the lung (Figure 7C) and small intestine (Figure 7D) of homozygous Cftr ${ }^{\text {F508del }}$ mice. Moreover, anakinra increased membrane expression of p.Phe508del-CFTR on p.Phe508del-CFTR-transfected CFBE410- cells as revealed by immunofluorescence staining (Figure 7E). Immunoblots (Figure $8 \mathrm{~A}$ ) revealed that at $37^{\circ} \mathrm{C}$, anakinra induced the core glycosylated band $\mathrm{B}$ to a greater extent than the mature band C of CFTR, an effect suggesting the occurrence of the unconventional secretory pathway that, bypassing the Golgi, takes immature CFTR directly at the plasma membrane (35). Indeed, anakinra promoted secretory autophagy involving GRASP55, a protein mediating unconventional 
A
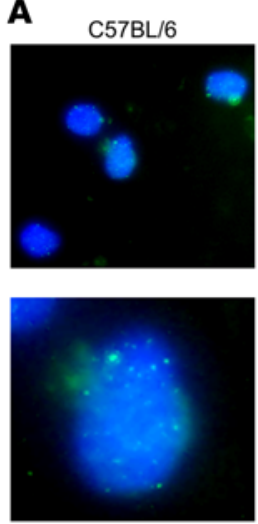

DAPI Anakinra

D
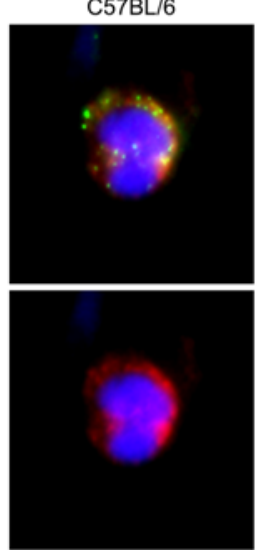

DAPI AhR Anakinra
B
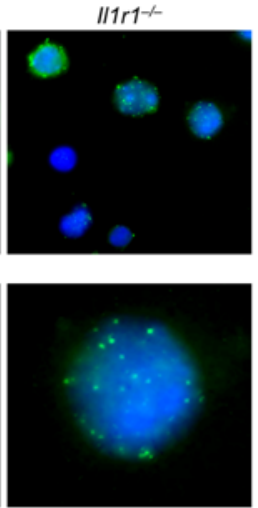

.

E
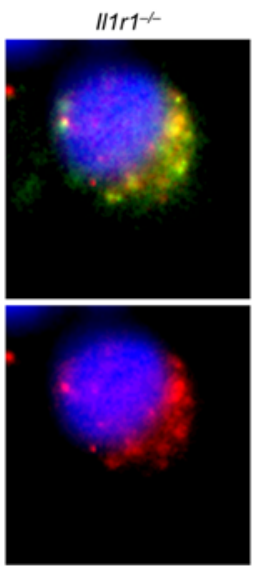

$30 \mathrm{~min}$

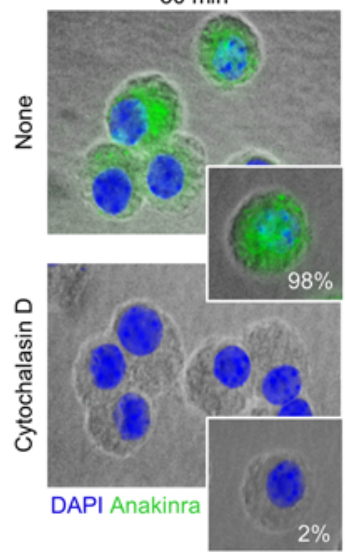

E Anakinra
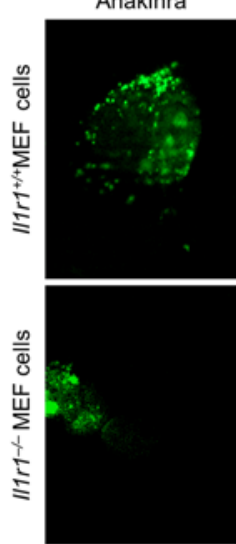
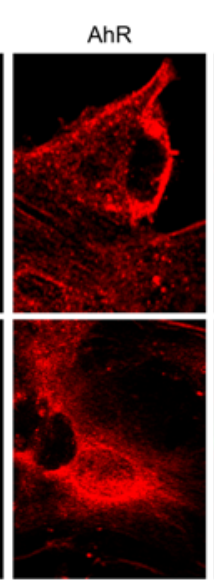

$60 \mathrm{~min}$

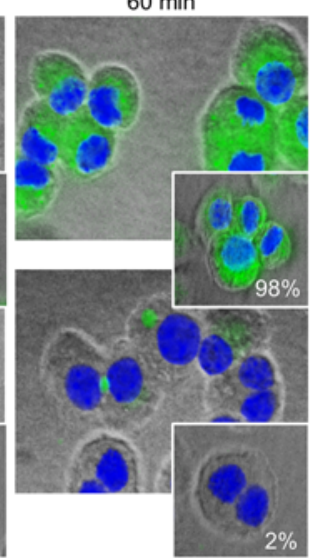

Merge

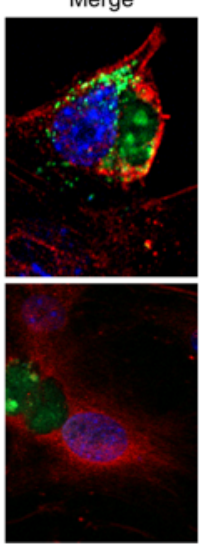

C

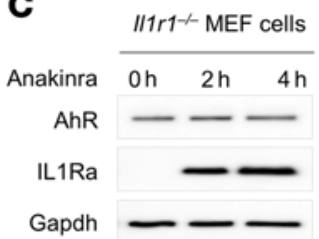

$\mathbf{F}$

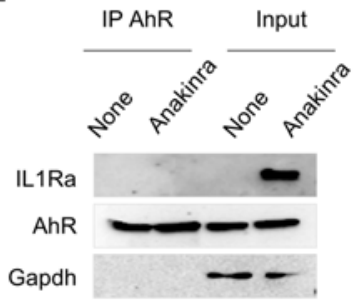

Figure 4. Anakinra colocalizes with AhR in II1rt/- cells. (A) Immunofluorescence imaging of cellular localization of FITC-anakinra in alveolar macrophages, isolated from naive C57BL/6 or $117 \mathrm{r}^{1 /-}$ mice, primed with $100 \mathrm{ng} / \mathrm{mL} \mathrm{LPS} \mathrm{for} 2$ hours at $37^{\circ} \mathrm{C}$, and exposed to $10 \mu \mathrm{g} / \mathrm{mL}$ FITC-anakinra for $60 \mathrm{minutes}$. (B) Immunofluorescence analysis of FITC-anakinra in II1r1-/- cells exposed to FITC-anakinra at 30 and 60 minutes in the presence of $5 \mu \mathrm{M}$ cytochalasin D. Insets, percentage positive cells. (C) $1 / 17 r^{-1-}$ MEF cells were treated with $10 \mu \mathrm{g} / \mathrm{mL}$ anakinra for 0,2 , and 4 hours and assessed for AhR and IL1Ra expression by immunoblotting with specific antibodies. (D) Immunofluorescence imaging of alveolar macrophages from naive $\left[57 \mathrm{BL} / 6\right.$ and $/ 17 \mathrm{r}^{-1-}$ mice primed with $100 \mathrm{ng} / \mathrm{mL}$ LPS for 2 hours at $37^{\circ} \mathrm{C}$, exposed to $10 \mu \mathrm{g} / \mathrm{mL}$ FITC-anakinra for 60 minutes, and stained with anti-AhR antibody. (E) Immunofluorescence imaging of MEF cells stained with anti-AhR antibody and DAPI and exposed to $10 \mu \mathrm{g} / \mathrm{mL}$ FITC-anakinra for 60 minutes. (F) $/ 17 r^{-/-}$MEF cells were treated with $10 \mu \mathrm{g} / \mathrm{mL}$ anakinra, lysed, immunoprecipitated with anti-AhR antibody, and assessed for IL1Ra and AhR expression by immunoblotting with specific antibodies. None, untreated cells. Representative images, acquired with a fluorescent microscope (BX51), and immunoblots from 2 independent experiments are shown. DAPI was used to detect nuclei. Sections were examined using a Zeiss Axio Observer Z1.

secretion of CFTR in the condition of ER stress blockade (35). Figure $8 \mathrm{~B}$ shows that colocalization of CFTR with GRASP55 was greatly promoted by anakinra and brefeldin A and greatly reduced upon GRASP55 inhibition by SiRNA. The rescuing activity of anakinra correlated with an increased chloride ion channel function of the rescued p.Phe508del-CFTR to an extent similar to that obtained with the corrector VX-809 (Figure 9, A and B). In a more relevant setting, anakinra increased the chloride channel activity in $\mathrm{HBE}$ cells from patients homozygous for the p.Phe508del-CFTR mutation. Ussing chamber tracings revealed that anakinra promoted, although to a variable degree, the forskolin-induced increase of the chloride current (Isc) in 3 out of 4 patients and not in the control group (Figure 9C), an effect that was sensitive to CFTR inhibition.

\section{Discussion}

The proteostasis network has established a new paradigm for deconvolution of disease mechanism and therapeutic management $(2,4)$.
However, since targeting all the myriad defects individually could be quite challenging, identifying a druggable pathway that controls disease-promoting processes may overcome the targeted delivery that is a critical factor influencing efficacy. This study showed that anakinra paired with xenobiotic-induced redox signaling to promote autophagy and proteostasis. As such, the study represents a proof-of concept demonstration of how the knowledge of proteostatic pathways directing lung function may improve opportunities for strategies to reduce lung injury and disease, as already pointed out (6). While not acting as a direct AhR ligand, anakinra promoted the generation of intracellular AhR ligands, such as kynurenine, via IDO1, whose activity was promoted via transcriptional and posttranscriptional mechanisms. Both mechanisms are supported by the ability of anakinra to inhibit IL- 6 and SOCS3, both driving proteasomal degradation of IDO1 (30), in mice (this study) and in humans (36) and by the ability of AhR to activate IDO1 transcription (37). Thus, the regulation of IDO1 degradation could be the 

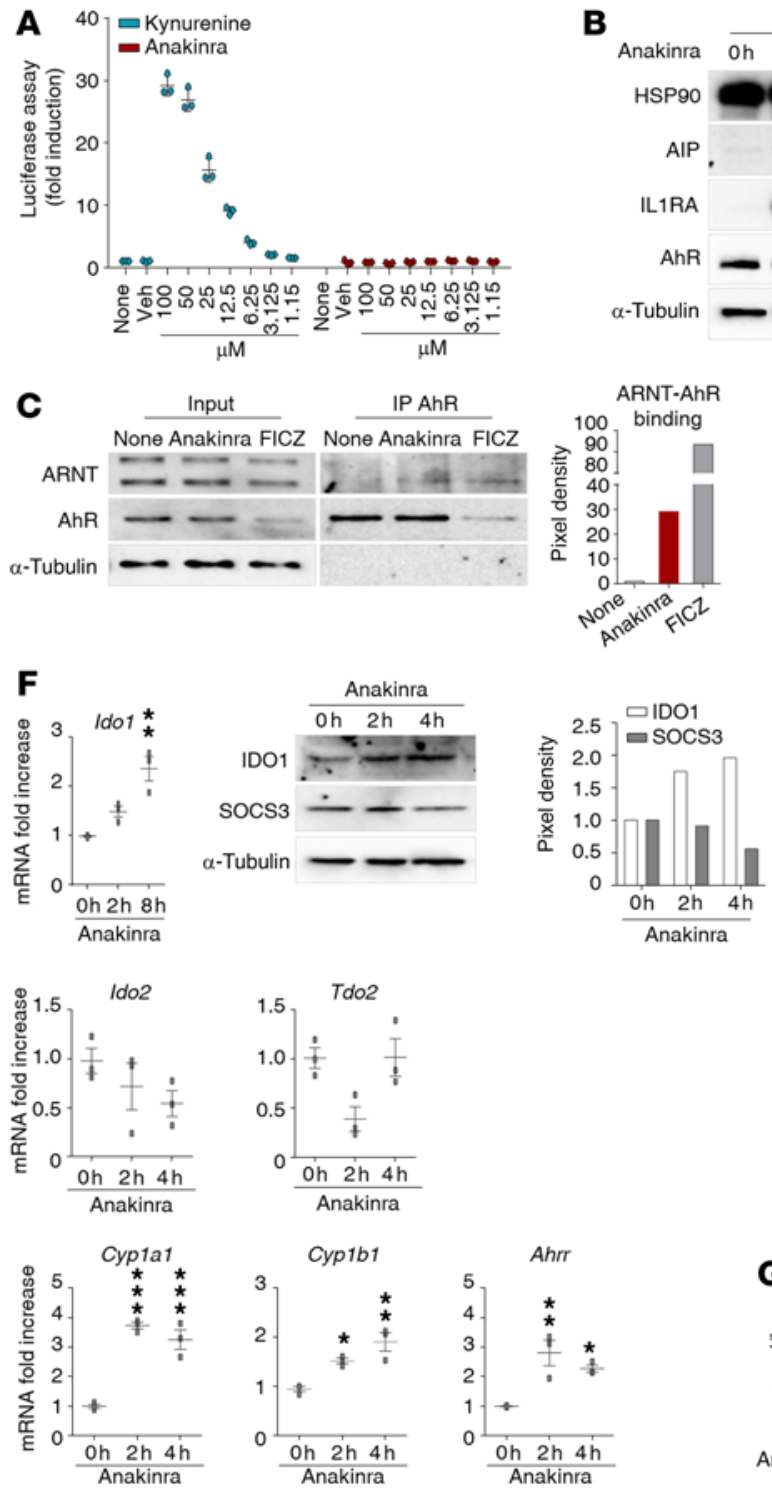
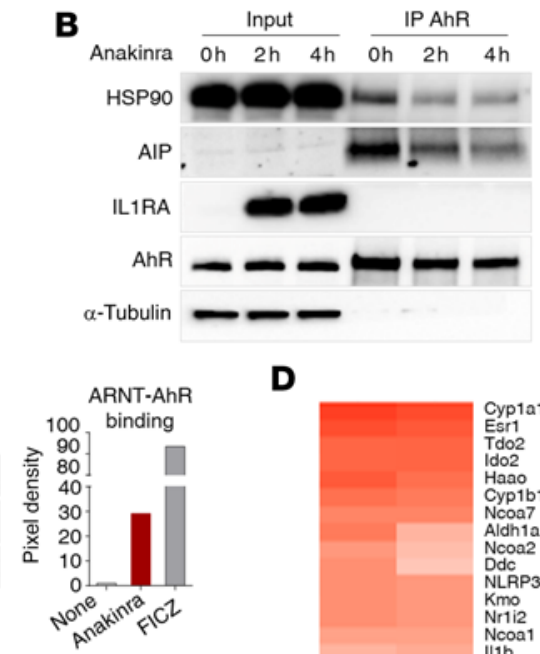

D
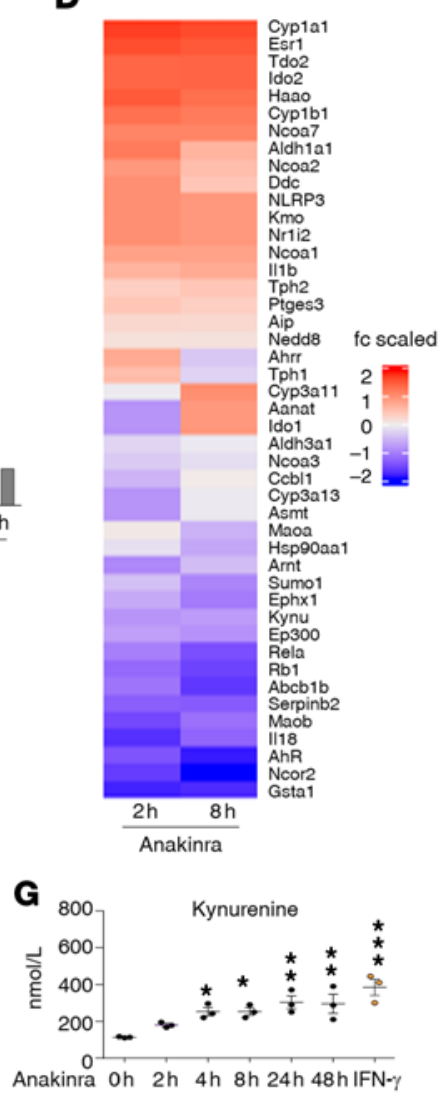
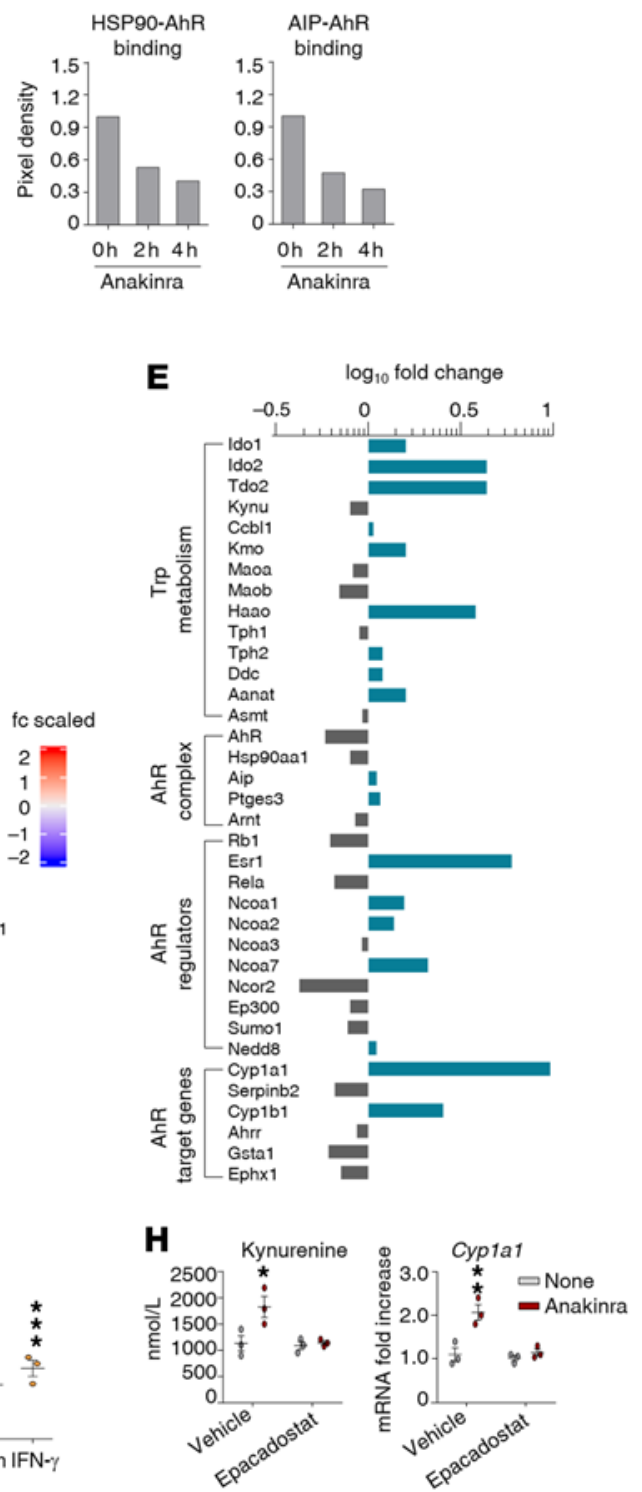

Figure 5. Anakinra activates a xenobiotic sensing pathway via ID01. (A) H1L1 cells were treated with different doses of kynurenine and anakinra for 6 hours and assessed for luciferase assay. (B) Representative immunoblots of ILIRA, HSP90, AIP, and AhR and (C) ARNT and AhR in cell lysates in which AhR was immunoprecipitated from $/ 11 \mathrm{r}^{-/-}$MEF cells treated with $10 \mu \mathrm{g} / \mathrm{mL}$ anakinra or $10 \mu \mathrm{M} \mathrm{FICZ}$. In B and C, data are representative of 1 out of 2 independent experiments and the relative densitometric analysis are reported. ( $D$ and $\mathbf{E}$ ) $11177^{-1-}$ MEF cells were treated with $10 \mu \mathrm{g} / \mathrm{mL}$ anakinra for 2 and 8 hours and analyzed for gene expression by a custom QuantiGene plex gene expression assay. Fold changes are reported as heatmap for 2 and 8 hours (D) and histograms for 8 hours (E) (data are representative of 1 out of 2 independent experiments). (F) $/ 11 r^{-1-}$ MEF cells were treated with $10 \mu \mathrm{g} / \mathrm{mL}$ anakinra and analyzed for mRNA expression of selected genes by RT-PCR ( $n=3$ independent samples) and protein expression of IDO1 and SOCS3 by immunoblotting (representative experiment). The relative densitometric analysis is reported. (G) $/ 11 r^{1 /-}$ MEF cells were treated with either $10 \mu \mathrm{g} / \mathrm{mL}$ anakinra for different times or $10 \mathrm{ng} / \mathrm{mL}$ IFN- $\gamma$ as positive control for 48 hours and assessed for kynurenine production by ELISA. (H) I/1r1 $7^{-/-}$MEF cells were treated with 10 $\mu \mathrm{g} / \mathrm{mL}$ anakinra in the presence or absence of $10 \mu \mathrm{M}$ epacadostat and assessed for kynurenine production by ELISA and Cyp1a1 gene expression by RT-PCR ( $n=3$ independent samples). ${ }^{*} P<0.05,{ }^{* *} P<0.01,{ }^{* *} P<0.001$, treated versus untreated (None or $0 \mathrm{~h}$ ) cells. One-way ANOVA, Bonferroni post hoc test.

initial working mechanism of anakinra that subsequently exploits the positive IDO1/kynurenine/AhR feedback loop that may limit inflammation in the condition of IL-1R1 deficiency. The finding that both kynurenine level (Supplemental Table 3) and AhR expression (Supplemental Figure 11) were increased in the condition of IL-1R1 deficiency supports this conclusion.

AhR transcriptionally activates NOX4, a critical mediator of autophagy via $\mathrm{H}_{2} \mathrm{O}_{2}(34)$. Besides the ER, NOX4 also localizes to the inner mitochondria membrane, where it functions as energetic sensor in this organelle (38). The mitochondrial localization of NOX4 is of interest because anakinra is known to induce mitochondrial SOD2 among the $\mathrm{H}_{2} \mathrm{O}_{2}$ scavengers (39). Given that SOD2 protects against mitochondrial oxidative damage and mitochondrial dysfunction (40), this implies that anakinra, by acting as a mitochondria-targeted antioxidant agent, can protect against oxidant- and mitochondrial-related disorders. Thus, the promotion of autophagy through a redox-signaling pathway involving mitochondrial NOX4 activity is a potentially novel mechanism through which 
A
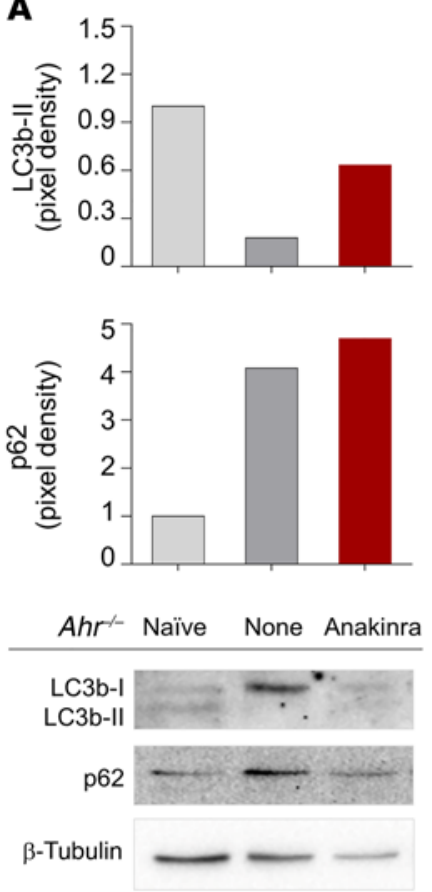

E
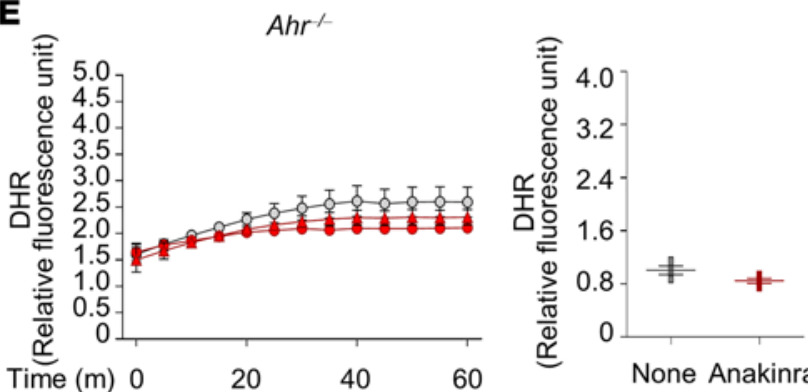

O None $\bullet$ Anakinra $\Delta$ Anakinra + MITO

G
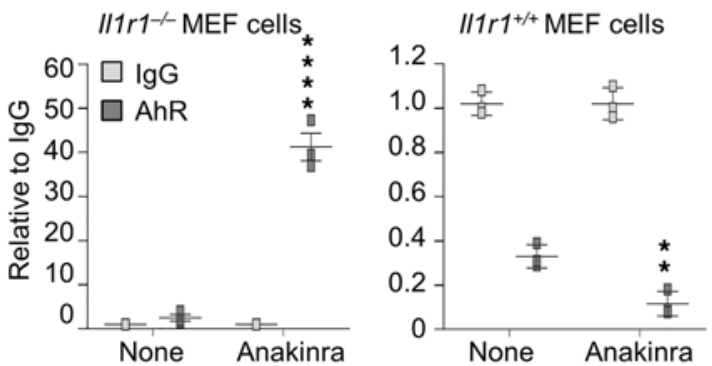
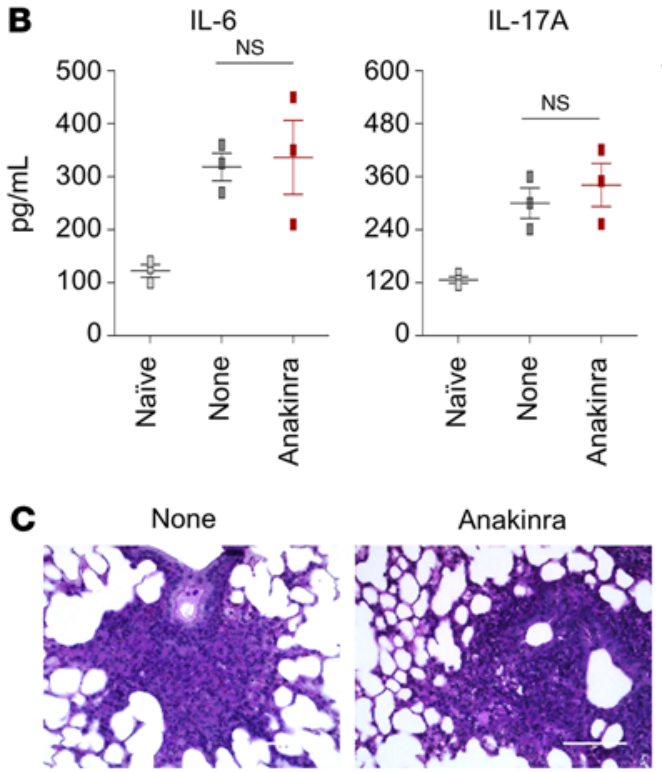

IL-1 $\beta$
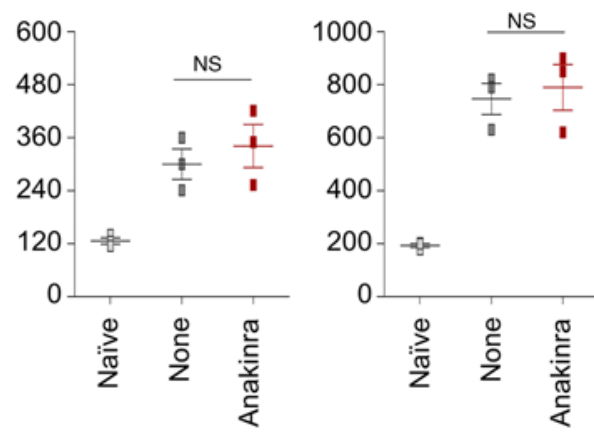
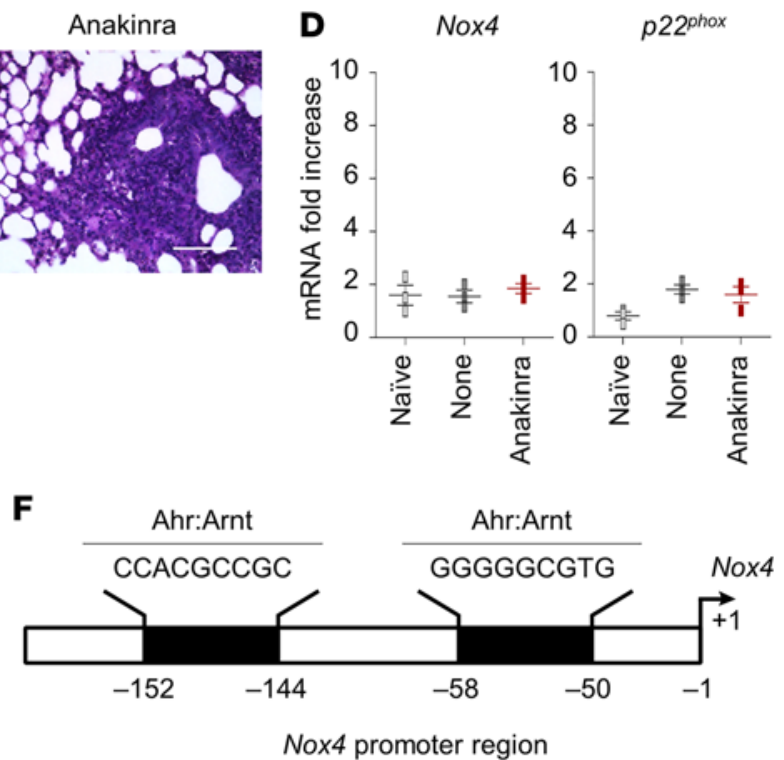

Figure 6. Anakinra promotes AhR transcriptional activity. (A-C) $A h R^{-/-}$mice were infected and treated with anakinra as in legend to Figure 1 and assessed for immunoblotting of LC3b and p62 (A), cytokine production (ELISA) in lung homogenates (B), and lung histology (PAS staining) at 7 days after infection (C). Scale bar: $200 \mu \mathrm{m}$. Data represent 3 independent experiments. Each in vivo experiment includes 6 to 8 mice per group, pooled before analysis. NS, not statistically significant, treated versus untreated (None) cells. One-way ANOVA, Bonferroni post hoc test. ( $\mathbf{D}$ and $\mathbf{E})$ Ex vivo purified alveolar macrophages and total lung cells from $\mathrm{Ahr}^{-1}$ mice infected and treated with anakinra were assessed for Nox4 and $p 22^{\text {phox }}$ expression (RT-PCR) ( $n=3$ independent samples) (D) and $\mathrm{H}_{2} \mathrm{O}_{2}$ production (DHR staining) ( $n=2$ independent samples) (E). (F) Illustration of predicted binding sites from the ALGGEN-PROMO database and the Eukaryotic Promoter Database. (C) $117 r^{-/-}$and $I 11 r r^{+/+}$MEF cells were treated with $10 \mu \mathrm{g} / \mathrm{mL}$ anakinra. ChIP assay was performed with AhR antibody. IgG was used as negative control. qPCR was conducted at the promoter regions of Nox4. Data are technical replicates of 1 representative out of 2 independent experiments.

anakinra $(14,41)$. Thus, within the emerging therapeutic option of targeting the NLRP3 inflammasome as antiinflammatory strategy in $\mathrm{CF}$, the finding that anakinra could have additional beneficial effects beyond NLRP3 inhibition (17) and IL-1 blockade (42) further reinforces its repurposing as a therapeutic agent in CF. This is emphasized by a recent trial that will investigate the effects of anakinra in CF (ClinicalTrials.gov NCT03925194).

By showing the involvement of the xenobiotic sensing AhR pathway, this study provides further foundations for the therapeutic potential of anakinra. Although the extent to which the IL-1R1- 


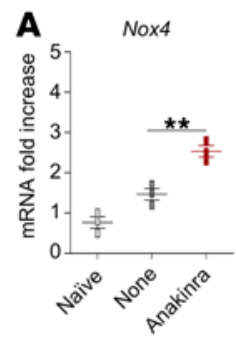

D

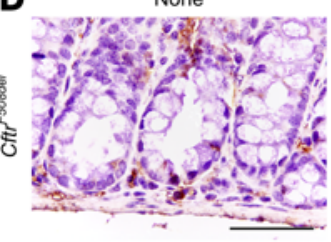

E

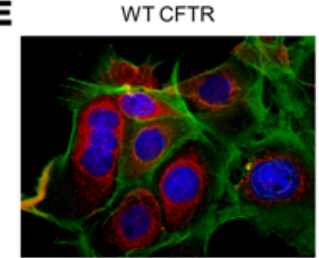

DAPI CFTR Phalloidin
B

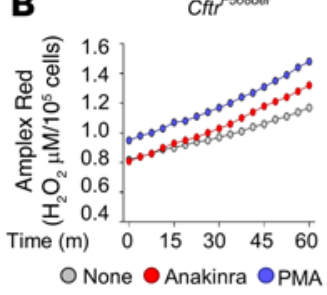

C
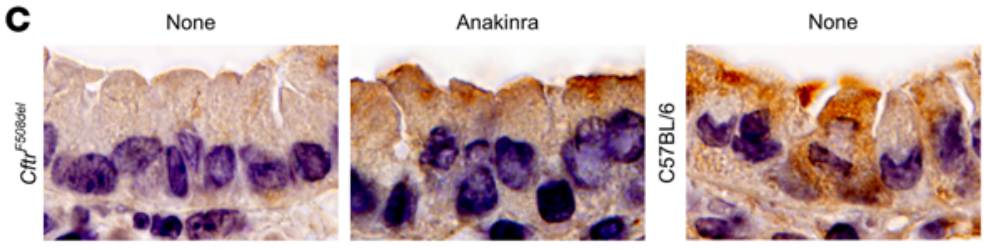

Anakinra

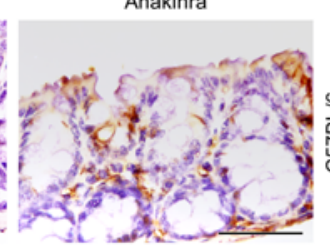

p.Phe508del-CFTR

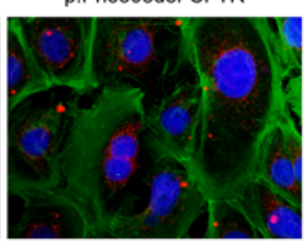

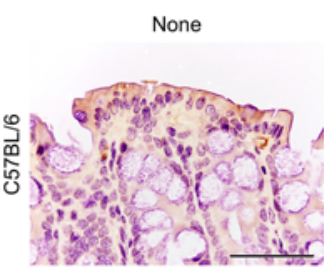

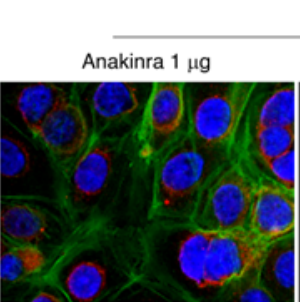

p.Phe508del-CFTR

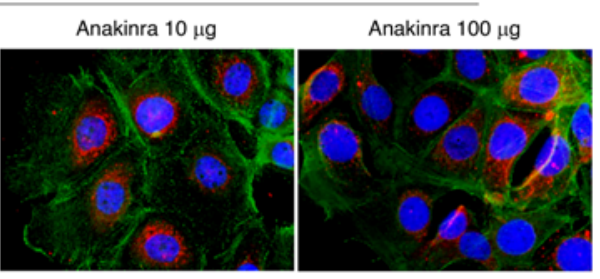

Figure 7. Anakinra restores cellular proteostasis in CF. (A-D) Cftr ${ }^{\mathrm{F} 58 \mathrm{ddel}}$ mice were infected (i.n.) with live $A$. fumigatus conidia and treated with anakinra 10 $\mathrm{mg} / \mathrm{kg}$ (i.p.) for 6 days. (A) Nox4 gene expression in total lung cells at $7 \mathrm{dpi}$ ( $n=3$ independent samples). None: infected, untreated mice. ${ }^{* *} P<0.01$, anakinra-treated versus untreated (None) mice. One-way ANOVA, Bonferroni post hoc test. (B) Amplex red fluorescence in purified alveolar macrophages from Cftr ${ }^{F 508 d e l}$ mice after stimulation with $10 \mu \mathrm{g} / \mathrm{mL}$ anakinra or $10 \mathrm{ng} / \mathrm{mL}$ PMA for 4 hours at $37^{\circ} \mathrm{C}$. For $P$ values, see Supplemental Figure 5 . Two-way ANOVA, Bonferroni post hoc test. (C) Lung and (D) small intestine expression of CFTR in mice infected and treated as above by IHC staining with anti-CFTR CF3 antibody (scale bar: $200 \mu \mathrm{m}$ ). Sections are representative of 3 independent experiments with $n=6$ mice per group. C57BL/ 6 mice are shown as control. Images were acquired high-resolution microscopy (Olympus DP71 using $\times 100$ objective). (E) Immunofluorescence staining of CFTR in CFBE410- cells stably transfected with WT or mutant CFTR and treated with different doses of anakinra for 4 hours at $37^{\circ} \mathrm{C}$. Staining was done with the anti-CFTR CF3 antibody followed by Alexa Fluor 555 and Alexa Fluor 488 anti-phalloidin for F-actin labeling. Data are representative of 1 out of 2 (E) or 3 (A-D) independent experiments.

dependent and independent pathways are intertwined in response to anakinra in vivo is likely dependent on many factors, including the levels of expression of IL1R1 and AhR, and the specific cell type, the different intracellular pathways activated by anakinra may account for its multifaceted activity and off-target effects and predict the contingency of anakinra's effects on environment and epigenetic factors affecting AhR activity. In line with our findings, a recent study suggested that IL-1Ra pairs with xenobiotic-induced immune signaling in human disease (43). Thus, at the intersection of mitochondrial oxidative stress, autophagy, and proteostasis, anakinra could be exploited in a variety of chronic human diseases associated with dysregulated redox status, defective proteostasis, and autophagy, such as aging and COVID-19 $(44,45)$.

In conclusion, this study provides further foundations for the possible use of anakinra in lung inflammatory diseases and suggests that the repurposing of anakinra at the intersection of mitochondrial oxidative stress, autophagy, and proteostasis in human diseases warrants further investigation.

\section{Methods}

Mice, infections, and treatments. Male and female, 8- to 10-week-old, C57BL6 WT mice were purchased from the Jackson Laboratory. Breeding pairs of homozygous $\mathrm{Illr}^{-{ }^{--}}$(Charles River Laboratories) and Ahr/- mice raised on C57BL6 background were bred under specific pathogen-free conditions at the breeding facilities of the University of Perugia, Perugia, Italy. B6.129-Ahrtm1Bra/J Ahr-deficient (Ahr-/) mice were supplied by B. Stockinger (MRC National Institute for Medical Research, London, United Kingdom). CF mice homozygous for the Phe508del-Cftr allele, which had been backcrossed for 12 generations to the C57BL/6 strain (Cftr ${ }^{\text {tm1Eur }}$ Phe508del, abbreviated Cftr ${ }^{\text {F508del }}$ ), were obtained from B. Scholte (Erasmus Medical Center, Rotterdam, Netherlands; ref. 46). These mice were provided with a special food consisting of an equal mixture of SRM-A (Arie Blok, Woerden) and Teklad 2019 (Harlan Laboratories) and water acidified to $\mathrm{pH} 2.0$ with $\mathrm{HCl}$ and containing $60 \mathrm{~g} / \mathrm{L}$ PEG 3350, $1.46 \mathrm{~g} / \mathrm{L} \mathrm{NaCl}, 0.745 \mathrm{~g} / \mathrm{L} \mathrm{KCl}$, $1.68 \mathrm{~g} / \mathrm{L} \mathrm{NaHCO}_{3}$, and $5.68 \mathrm{~g} / \mathrm{L} \mathrm{Na}_{2} \mathrm{SO}_{4}$. Newborn mice were genotyped by cutting a small piece of tail 12 days after birth. Mice were anesthetized in a plastic cage by inhalation of $3 \%$ isoflurane (Forane, Abbott) in oxygen before i.n. instilling $2 \times 10^{7}$ A. fumigatus (Af293) resting conidia per $20 \mu \mathrm{L}$ of saline. For P. aeruginosa infection, clinical $P$. aeruginosa strain, isolated from a patient, was obtained from the Diagnostic Unit of Microbiology from the University of Perugia. The bacteria were grown for 3 hours to reach the exponential phase. Next, the bacteria were pelleted by centrifugation $(2700 g, 15$ minutes) and washed twice with sterile PBS, and the OD of the bacterial suspension was adjusted by spectrophotometry at $600 \mathrm{~nm}$. The intended number of CFUs was extrapolated from a standard growth curve. Appropriate dilutions with sterile PBS were made to prepare the inoculum before 

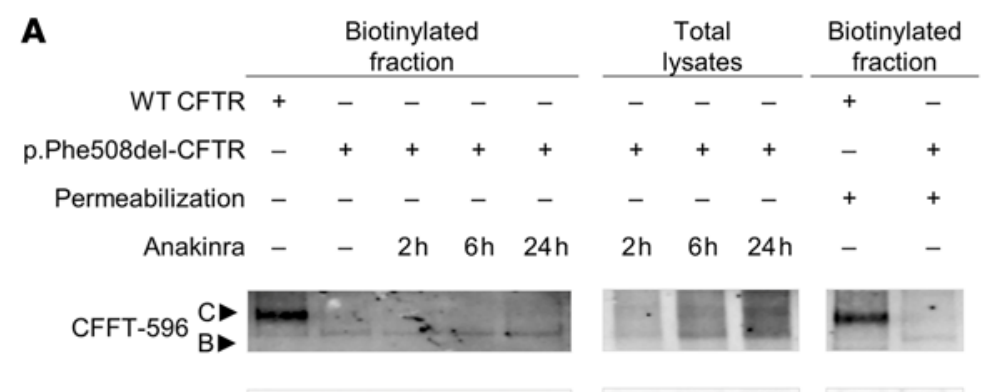

Calnexin

B

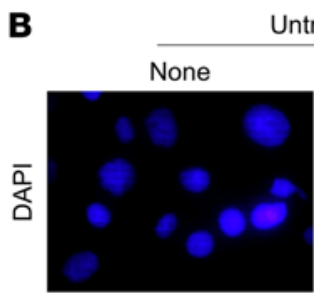

Untreated
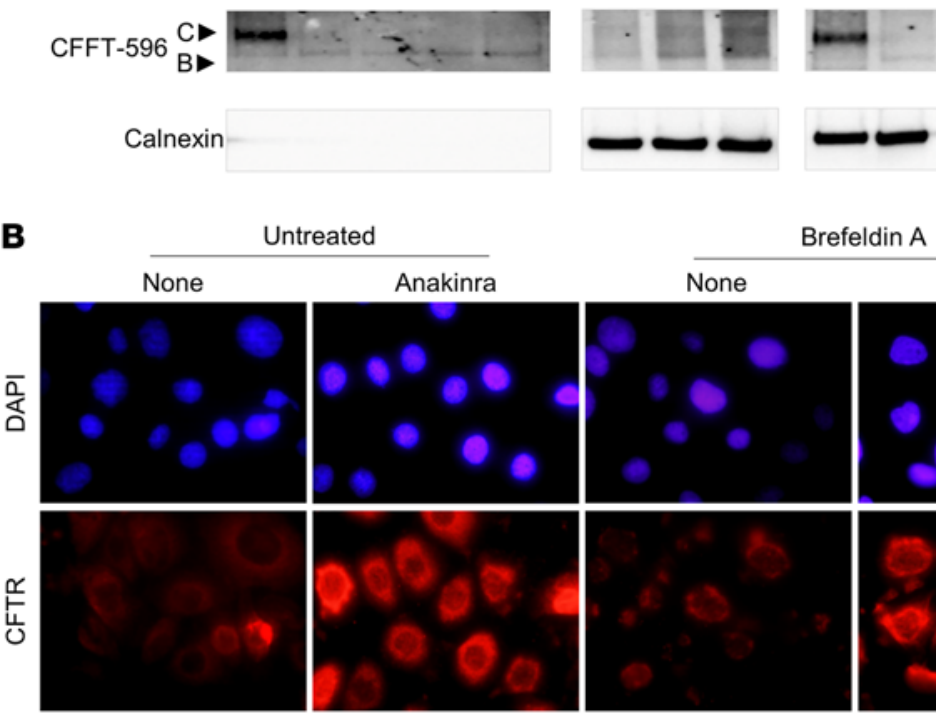

Brefeldin A
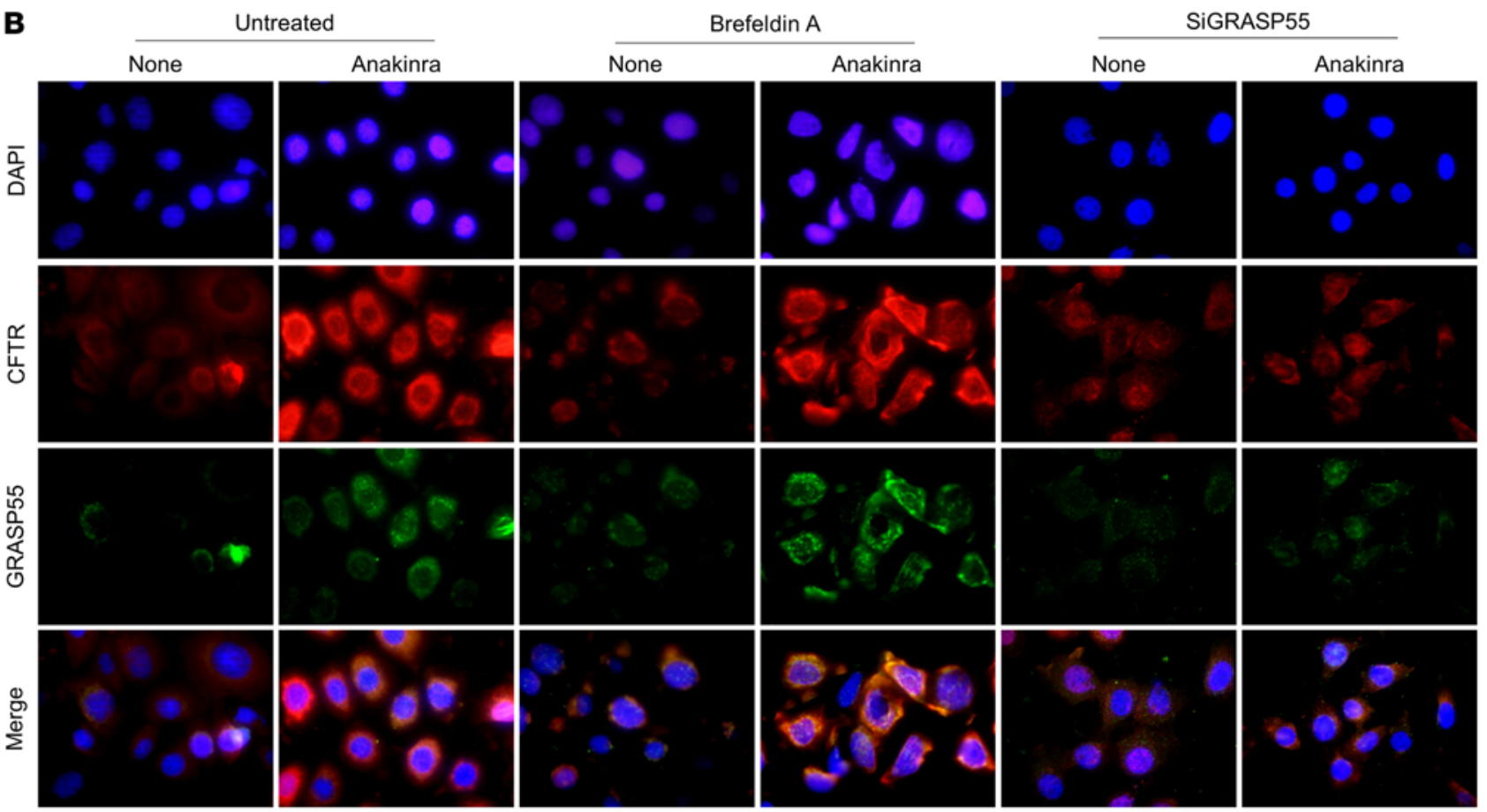

Figure 8. Anakinra increases surface expression of p.Phe508del-CFTR via the unconventional secretory pathway. Cell surface expression of CFTR in HEK293 cells transiently transfected with HA-p.Phe508del-CFTR and HA-WT-CFTR pCDNA3.1 plasmids and treated for 2, 6, or 24 hours with $10 \mu \mathrm{g} / \mathrm{mL}$ of anakinra at $37^{\circ} \mathrm{C}$ (A). Cells were immunoblotted with the anti-CFTR 596 and anti-calnexin antibodies after cell surface biotinylation and incubation with avidin solution. (B) Immunofluorescence staining of CFTR and CRASP55 in p.Phe508del-CFTR-transfected CFBE410- cells treated with $10 \mu \mathrm{g} / \mathrm{mL}$ anakinra or vehicle (None) at $37^{\circ} \mathrm{C}$. Cells were pretreated with GRASP55 SiRNA or brefeldin A for 24 or 6 hours, respectively, at $37^{\circ} \mathrm{C}$. Nuclei were counterstained with DAPI. Data are representative of 3 independent experiments.

i.n. instilling $3 \times 10^{7} \mathrm{CFU}$ per mice. Quantification of fungal growth was done as described (17). Bronchoalveolar lavage (BAL) fluid was collected in a plastic tube on ice and centrifuged at $400 \mathrm{~g}$ at $4^{\circ} \mathrm{C}$ for 5 minutes. For differential BAL fluid cell counts, cytospin preparations were made and stained with May-Grünwald Giemsa reagents (Sigma-Aldrich). For histology, paraffin-embedded sections were stained with periodic acid-Schiff (PAS). Mice were treated (i.p.) with $10 \mathrm{mg} / \mathrm{kg}$ anakinra reconstituted in sterile water daily for 6 consecutive days beginning the day of the infection. Mice were euthanized a day after treatment. Infections were performed under isoflurane anesthesia, and all efforts were made to minimize suffering.

Cells and treatments. RAW 264.7 cells were from ATCC (TIB71). MEF cells were generated as described (47). HEK293 cells were from ATCC (CRL-1573). HBE cells, homozygous for the p.Phe508del mutation, and isogenic WT cells were obtained from lung transplants (individuals with $\mathrm{CF}$ ) or lung resections (control) (provided by $\mathrm{LJ}$
Galietta, Italian Cystic Fibrosis Foundation, Verona, Italy). Cells were maintained at $37^{\circ} \mathrm{C}$ in a humidified incubator in an atmosphere containing $5 \% \mathrm{CO}_{2}$. Stable lentiviral-based transductions of the parental CFBE410 ${ }^{-}$cells, homozygous for the p.Phe508del-CFTR mutation (48), with either WT CFTR or p.Phe508del-CFTR, were provided by LJ Galietta. The transduced CFBE410 cells were maintained in MEM supplemented with $50 \mathrm{U} / \mathrm{mL}$ penicillin, $50 \mu \mathrm{g} / \mathrm{mL}$ streptomycin, $2 \mathrm{mM}$ L-glutamine, $10 \%$ FBS, and $1 \mu \mathrm{g} / \mathrm{mL}$ blasticidin (WT CFTR) or $2 \mu \mathrm{g} / \mathrm{mL}$ puromycin (p.Phe508del-CFTR) in a 5\% $\mathrm{CO}_{2}$ and $95 \%$ air incubator at $37^{\circ} \mathrm{C}$. To establish polarized monolayers, CFBE410 ${ }^{-}$cells were seeded on $24 \mathrm{~mm}$-diameter Transwell permeable supports $(0.4 \mathrm{~mm}$ pore size; Corning Corp) at $2 \times 10^{6}$ cells/well and grown in an air-liquid interface culture at $37^{\circ} \mathrm{C}$ for $6^{-9}$ days and then at $27^{\circ} \mathrm{C}$ for 36 hours. Fisher rat thyroid (FRT) epithelial cells, provided by LJ Galietta, were cultured on 60 $\mathrm{mm}$ Petri dishes with Coon's modified F12 containing 5\% serum, $2 \mathrm{mM}$ L-glutamine, $50 \mathrm{U} \mathrm{mL}^{-1}$ penicillin, and $50 \mu \mathrm{g} \mathrm{mL}^{-1}$ streptomycin. Cells 
A

Fsk $(10 \mathrm{mM})+\operatorname{Gen}(30 \mathrm{mM})$ CFTR inh-172 (1 mM)

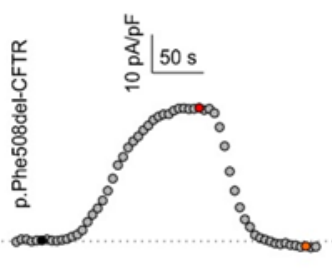

None

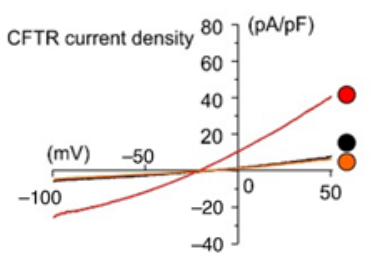

Fsk $(10 \mathrm{mM})+$ Gen $(30 \mathrm{mM})$

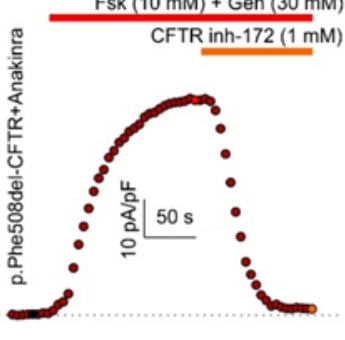

Anakinra

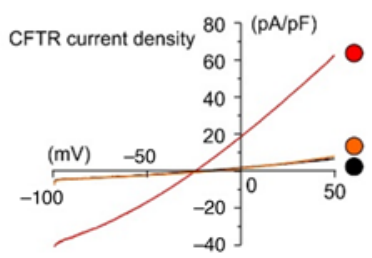

$F s k(10 \mathrm{mM})+\operatorname{Gen}(30 \mathrm{mM})$

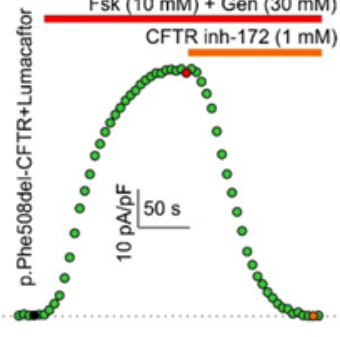

VX-809

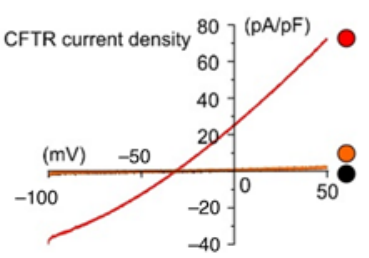

B

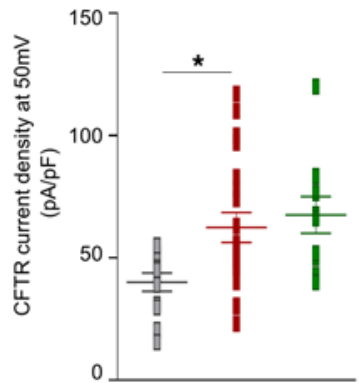

p.Phe508del-CFTR

C Control

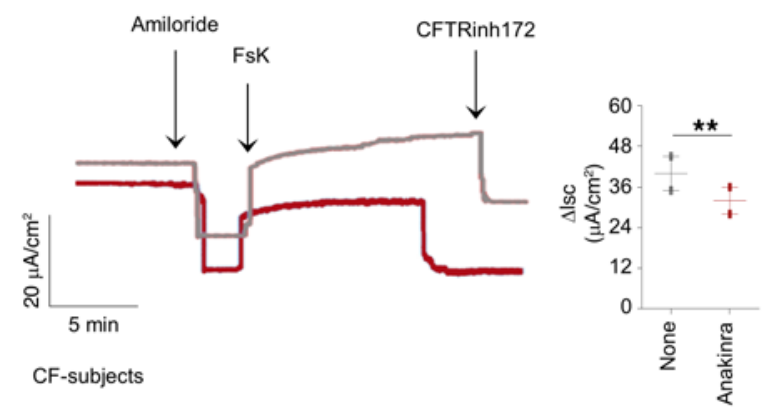

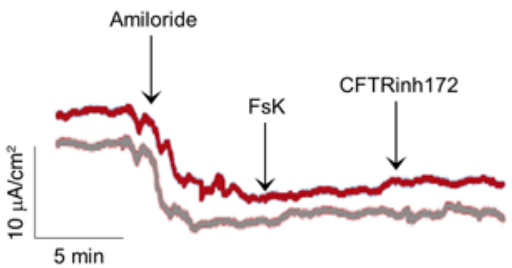

CFTRinh172

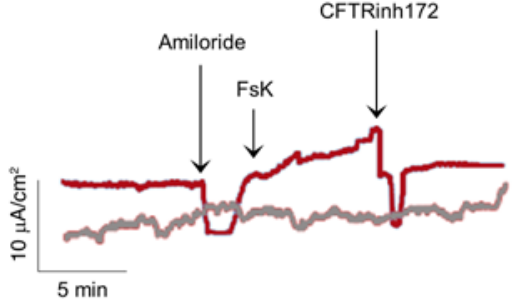

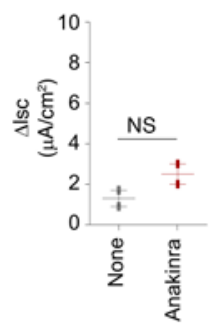

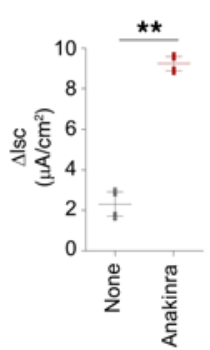

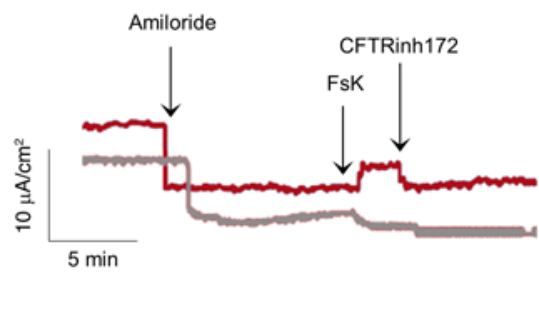
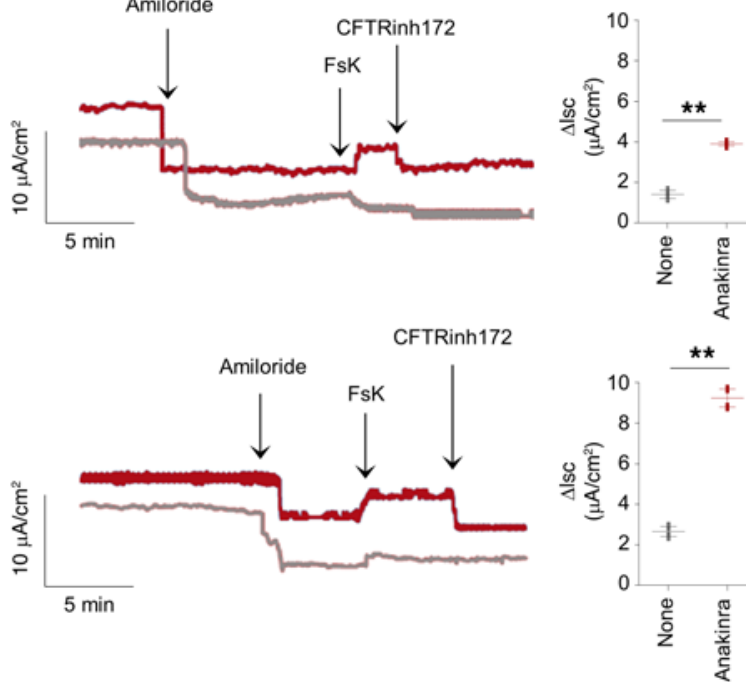

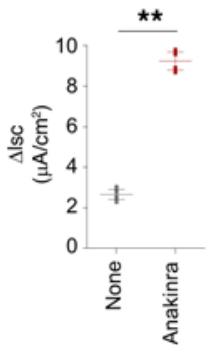

Figure 9. Anakinra increases CFTR activity. (A) Time course of whole-cell CFTR current densities induced by $10 \mu \mathrm{M}$ forskolin (Fsk) $+30 \mu \mathrm{M}$ genistein (Cen)

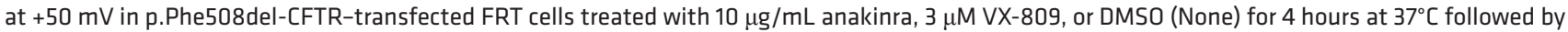
blockade with CFTR inhibitor 172 (CFTR inh-172). The horizontal bars indicate the time period of drug application. Inset, current ramps from -100 mV to $+50 \mathrm{mV}$ (holding potential, $-40 \mathrm{mV}$ ) in control condition (black trace), after application of Fsk + Gen (red trace), and after application of $1 \mu \mathrm{M}$ CFTRinh-172 (orange trace). (B) Mean \pm SEM CFTR current density measured at $+50 \mathrm{mV}$ induced by Fsk and Gen in cells treated with anakinra $(n=20)$, VX-809 $(n=10)$, or vehicle $(n=12) .{ }^{*} P<0.05$, anakinra versus untreated (None), 1-way ANOVA, Bonferroni post hoc test. (C) CFTR-dependent chloride secretion measured by means of Fsk-induced increase in the Isc in HBE cells from 4 patients with CF and 1 representative control treated with anakinra for 4 hours at $37^{\circ} \mathrm{C}$ and mounted in Ussing chambers in the presence of CFTR inhibition (CFTR inh-172) and amiloride. ${ }^{* *} P<0.01$, anakinra-treated versus untreated (None) cells. NS, not statistically significant. Student's $t$ test. Data are from 2 experiments. 
stably transfected with CFTR plasmid were provided by LJ Galietta. Total lung cells and alveolar macrophages were obtained from C57BL6 and Illr $\mathrm{I}^{-/-}$mice as described (14).

Anakinra (Kineret, Amgen Europe) was diluted in PBS $1 \times$ (vehicle). Cells were incubated with different doses of anakinra for up to 24 hours. FITC-anakinra was obtained with the FITC Conjugation Kit (Abcam), as per the manufacturer's instructions. Truncated anakinra was obtained as described below. In selected experiments, cells were treated with the AhR agonists 5,11-dihydro-indolo[3,2-b]carbazole-6-carboxaldehyde, 6-formylindolo[3,2-b]carbazole (FICZ, Sigma-Aldrich), 2-(1'H-indole-3'-carbonyl)-thiazole-4-carboxylic acid (ITE, Sigma-Aldrich), and kynurenine (Sigma-Aldrich), or the IDO1 inhibitor epacadostat (SelleckChem).

Measurements of AhR activation. To assess the activation of AhR, we used mouse hepatoma cells (H1L1.1c2), containing the stably integrated AhR xenobiotic responsive element driven by a firefly luciferase reporter plasmid, pGudLuc6.1 (49). Cells were seeded in 96-well plates at a density of $0.1 \times 10^{6}$ cells in $200 \mu \mathrm{L}$. After 12 hours at $37^{\circ} \mathrm{C}$, cells were stimulated for 6 hours with increasing concentrations of kynurenine or anakinra before lysis. Luciferase assays were performed using luciferase reporter assay kit (Promega). The Renilla luciferase activity was measured, and results are presented as fold induction.

Limited proteolysis. The proteolytic cleavage of anakinra was performed as described (50). Briefly, a stock protein solution at $10 \mathrm{mg} / \mathrm{mL}$ concentration was diluted in PBS pH 7.0 at $1 \mathrm{mg} / \mathrm{mL}$ and incubated at $25^{\circ} \mathrm{C}$ with proteinase $\mathrm{K}$ (P5568, Sigma-Aldrich) at an enzyme/substrate ratio of 1:100 for 1 hour. The reaction was stopped by addition of PMSF at $2 \mathrm{mM}$ concentration. As a control, in a parallel sample, PMSF was added from the beginning. Protein cleavage was evaluated by SDS-PAGE analysis (Supplemental Figure 2A). The overall folding of cleaved anakinra was evaluated by registering intrinsic fluorescence emission spectra before and after proteinase $\mathrm{K}$ treatment using a Jasco J-715 spectropolarimeter equipped with a thermostatically controlled cell holder. Protein emission spectra were taken from 300 to $500 \mathrm{~nm}$ (excitation at $280 \mathrm{~nm}$ ) with both the excitation and the emission slits set to $5 \mathrm{~nm}$. All spectra were corrected by subtracting the emission spectrum of the blanks, i.e., of samples containing all reagents except anakinra (Supplemental Figure 2B).

Genome sequencing, assembly, and analysis. Total RNA was extracted from C57BL/6 and Illr1 ${ }^{-/}$alveolar macrophages purified and stimulated in vitro with $10 \mu \mathrm{g} / \mathrm{mL}$ of anakinra for 4 hours at $37^{\circ} \mathrm{C}$. The total RNA samples were first treated with DNase I to degrade any possible DNA contamination. Then, the mRNA was enriched by using oligo(dT) magnetic beads. Mixed with the fragmentation buffer, the mRNA was fragmented into short fragments (about $200 \mathrm{bp}$ ). Then, the first strand of cDNA was synthesized by using random hexamer-primers. Buffer, dNTPs, RNase H, and DNA polymerase I were added to synthesize the second strand. The double-strand cDNA was purified with magnetic beads. End reparation and 3 '-end single nucleotide A (adenine) addition was then performed. Finally, sequencing adaptors were ligated to the fragments. The fragments were enriched by PCR amplification. During the quality control step, Agilent 2100 Bioanalyzer and ABI StepOnePlus Real-Time PCR System were used to qualify and quantify of the sample library. The library products were ready for sequencing via Illumina HiSeq 2000 or another sequencer when necessary. Illumina sequencing using the HiSeq 2000 platform was performed at the Beijing Genomics Institute, Shenzhen, China (www.genomics.cn/index.php) according to the manufacturer's instructions. Functional annotation by GO (http:// www.geneontology.org) was analyzed by Blast2go (51) and WEGO (52) software. The gene expression data set has been deposited in NCBI's Gene Expression Omnibus database (accession number GSE188809).

Autophagy. RAW264.7 cells were treated with $50 \mu \mathrm{M}$ rapamycin (LC laboratories), A. fumigatus swollen conidia (1:1 ratio), and/or 10 $\mu \mathrm{g} / \mathrm{mL}$ anakinra, full-length and or truncated, at $37^{\circ} \mathrm{C}$ for 4 hours. In selected experiments, $100 \mathrm{nM}$ bafilomycin A1 (Sigma-Aldrich) or 100 $\mu \mathrm{M}$ chloroquine (Sigma-Aldrich) was added. After treatment, cells were fixed in $2 \%$ formaldehyde for 15 minutes at room temperature and permeabilized in blocking buffer containing 3\% BSA and $0.5 \%$ Triton X-100 in PBS. The cells were then incubated at $4^{\circ} \mathrm{C}$ with the primary antibody anti-LC3b (Abcam, ab48394). After extensive washing with PBS, the slides were incubated at room temperature for $60 \mathrm{~min}$ utes with goat anti-rabbit antibody to LC3 followed by Alexa Fluor 488 (Molecular Probes). DAPI (Molecular Probes) was used to counterstain nuclei. Optical sections were examined using a Zeiss Axio Observer Z1 inverted microscope equipped with ApoTome filter and Axiocam MRm camera detection system. The number of LC3 puncta was quantified with Image (NIH) (53) and expressed as number of puncta/cell. In selected experiments, RAW264.7 cells were seeded in $100 \mathrm{~mm}$ Petri dish $\left(3.5 \times 10^{6}\right)$ and transfected with the EGFP-LC3 plasmid (Addgene) using ExGen 500 in vitro transfection reagent (Fermentas) for 48 hours following the manufacturer's instructions. Transiently transfected RAW264.7 cells were exposed to A. fumigatus swollen conidia (1:1 ratio) and $10 \mu \mathrm{g} / \mathrm{mL}$ anakinra and incubated for 4 hours at $37^{\circ} \mathrm{C}$ in $5 \% \mathrm{CO}_{2}$ as described (17). Starvation was carried out in Earle's balanced salt solution. Cultures growing on coverslips were observed at $\times 100$ magnification with the Olympus BX51 fluorescence microscope using an FITC filter. For autophagy on lung cells, purified $1 \times 10^{6}$ alveolar macrophages from naive mice were stimulated on glass slides in 24 multi-well plates with anakinra and/or Aspergillus conidia as above for 4 hours at $37^{\circ} \mathrm{C}$ in $5 \% \mathrm{CO}_{2}$. Cells were incubated with 1:200 diluted anti-LC3 antibody (Cell Signaling Technology, 2775S) overnight at $4^{\circ} \mathrm{C}$ in PBS containing $3 \%$ normal BSA, incubated with anti-rabbit PE secondary antibody (Sigma-Aldrich), and fixed for 20 minutes in PBS containing 4\% paraformaldehyde. Images were acquired using the Olympus BX51 fluorescence microscope with a $\times 100$ objective and analySIS image processing software (Olympus). DAPI was used to detect nuclei.

SiRNA design and delivery. Predesigned SiRNA against Atg4a (MMC. RNAI.N174875.12.1), Nox4 (mm.Ri.Nox4.13.1), and GORASP2 (coding for GRASP55) (duplex name: hs.Ri.GORASP2.13.1) were purchased from Integrated DNA Technologies (IDT) (TEMA Ricerca). Cells were incubated for 24 hours (as indicated by preliminary experiments performed at 12,24 , or 48 hours) at $37^{\circ} \mathrm{C}$ in $5 \% \mathrm{CO}_{2}$ with specific SiRNA using Lipofectamine LTX reagent (Invitrogen) following the manufacturer's instructions. Effectiveness of silencing of specific targets was verified by real-time PCR (RT-PCR) analysis at 24 hours (Supplemental Figure 9). For in vivo experiments, each mouse received i.n. administration of $10 \mu \mathrm{g} / \mathrm{kg}$ unmodified SiRNA or equivalent dose of nonspecific control SiRNA duplex in a volume of $20 \mu \mathrm{L}$ of duplex buffer (IDT). I.n. SiRNA was given once the day before infection and 2 days after infection (54).

Western blot analysis and IP assay. For Western blot and IP assay, lung and cell lysates were harvested after treatment at the indicated time, washed twice with cold PBS (Sigma-Aldrich), and lysed in RIPA buffer (Tris/ $\mathrm{HCl}$ at $\mathrm{pH} 8.0,50 \mathrm{mM}, \mathrm{NaCl} 150 \mathrm{mM}$, SDS 0.1\%, sodium deoxycholate 1\%, Triton X-100 1\%), supplemented with protease inhibitor cocktail (PIC, Roche) and PMSF (Sigma-Aldrich). For IP, lysates were 
incubated overnight at $4^{\circ} \mathrm{C}$ with the specific antibody followed by 2 hours of incubation with protein G- or protein A-Sepharose beads (GE Healthcare). Immune complexes were washed 4 times in washing buffer and boiled in $2 \times$ sample buffer. The proteins were separated by electrophoresis on SDS-PAGE and detected using specific antibodies against LC3b I and II (Cell Signaling Technology, 2775S), p62 (Cell Signaling Technology, 5414S), IDO1 (cv152, as described in ref. 31), AhR (Invitrogen, MA1514), IL-1Ra (Invitrogen, PA5-21776), ARNT (Cell Signaling Technology, 5537), HSP90 (Proteintech, 13171-1-AP), AIP (Proteintech, 18176-1-AP), and SOCS3 (Cell Signaling Technology, 2923S). Normalization was performed with $\beta$-actin, $\alpha$-tubulin, $\beta$-tubulin, or Gapdh antibodies (Sigma-Aldrich, A3853, T9026, T4026, G8795). Protein signal intensities were quantified by densitometric analysis using Image J software. See complete unedited blots in the supplemental material.

ROS determination and fluorescence microscopy. For ROS determination, RAW 264.7 cells or ex vivo purified lung macrophages were plated in HBSS buffer with $\mathrm{Ca}^{2+}$ and $\mathrm{Mg}^{2+}$ and without phenol red and exposed to $10 \mu \mathrm{g} / \mathrm{mL}$ anakinra or $10 \mathrm{ng} / \mathrm{mL}$ PMA (phorbol 12-myristate 13-acetate, Sigma-Aldrich) for 4 hours at $37^{\circ} \mathrm{C}$. We used dihydroethidium (DHE, Molecular Probes) to detect intracellular and extracellular superoxide production by phagocytic NADPH oxidase (55); MitoSOX red (Molecular Probes) to detect mitochondrial superoxide; Amplex Red (Invitrogen) for $\mathrm{H}_{2} \mathrm{O}_{2}$; and DHR (Sigma-Aldrich) to detect intracellular ROS, mainly $\mathrm{O}_{2}-$ and $\mathrm{H}_{2} \mathrm{O}_{2}$ (56). As inhibitors, we used diphenyleneiodonium (DPI, Sigma-Aldrich) to inhibit NADPH oxidase, MitoTEMPO (Enzo Life Science) to scavenge mitochondrial ROS, and allopurinol (Sigma-Aldrich) to scavenge xanthine oxidase-dependent $\mathrm{O}_{2}-$ and $\mathrm{H}_{2} \mathrm{O}_{2}$. Next, $10 \mu \mathrm{M}$ DHR, $50 \mu \mathrm{M}$ DHE, and $5 \mu \mathrm{M}$ MitoSOX red were added to cells exposed to PMA or anakinra for the last 30 minutes at $37^{\circ} \mathrm{C}$, after which the reagents were washed out. Cells were preincubated with $50 \mu \mathrm{M}$ MitoTEMPO for 60 minutes before the addition of anakinra. Then, $50 \mu \mathrm{M}$ allopurinol or $2 \mu \mathrm{g} / \mathrm{mL}$ DPI was added for 20 or 60 minutes at $37^{\circ} \mathrm{C}$. The DHR (ex 100/ em 530), DHE (ex 518/em 605), and MitoSOX red (ex 510/em 580) signals were measured by a Tecan Infinite 200 microplate reader. During the measurement, the cells were maintained at $37^{\circ} \mathrm{C}$. For fluorescence microscopy, cells were fixed in PBS containing $4 \%$ paraformaldehyde. For colocalization, $\mathrm{H}_{2} \mathrm{O}_{2}$ was visualized using $1 \mathrm{mM}$ of DCF-DA (Molecular Probes), and mitochondria were stained with $200 \mathrm{nM}$ of MitoTracker red (Molecular Probes). Because $\mathrm{H}_{2} \mathrm{O}_{2}$ is the major peroxide in cells, it is generally accepted that DCF is proportional to $\mathrm{H}_{2} \mathrm{O}_{2}$ concentration (57). Images were acquired using the Olympus BX51 fluorescence microscope with either a $\times 100$ or $\times 40$ objective and analySIS image processing software (Olympus). For anakinra entry and AhR cellular localization, cells were exposed to $10 \mu \mathrm{g} / \mathrm{mL}$ FITC-anakinra for 30 minutes, and optical sections were examined using a Zeiss Axio Observer Z1 inverted microscope equipped with ApoTome filter and Axiocam MRm camera detection system after staining with rabbit anti-AhR antibody (Santa Cruz Biotechnology, sc-101104) or DAPI to detect nuclei.

ChIP assay. MEF cells were grown on $100 \mathrm{~mm}$ plates incubated with $10 \mu \mathrm{g} / \mathrm{mL}$ anakinra for 4 hours. Cells were then recovered in PBS $1 \times$ with $\mathrm{MgCl}_{2} 1 \mathrm{mM}$, exposed to $1 \%$ formaldehyde at room temperature for 15 minutes to induce DNA crosslinking, washed, and then lysed with RIPA buffer containing $150 \mathrm{mM} \mathrm{NaCl}, 50 \mathrm{mM}$ Tris $\mathrm{pH}$ 8.0, 1\% Triton X-100, $0.5 \%$ sodium deoxycholate, $0.1 \%$ PIC, and PMSF. Cell lysates were sonicated to shear DNA into 200 to 500 bp fragments and subjected to IP using ChIP-grade anti-AhR monoclonal antibody (Thermo Fisher Scientific, MA1-513) at $4^{\circ} \mathrm{C}$ overnight. Normal mouse IgG (Sigma-Aldrich,
12-371) was used as the ChIP-negative control. The ChIP product was incubated with salmon sperm conditioned protein A Sepharose for 3 hours at $4^{\circ} \mathrm{C}$ to recover AhR-bound DNA. The resulting material was washed; collected in CHIP elution buffer containing $300 \mathrm{mM} \mathrm{NaCl}, 10$ mM Tris pH 8.0, 0.5\% SDS, and 5 mM EDTA pH 8.0; and then de-crosslinked, purified, and resuspended in ultrapure water. AhR binding to chromatin was quantified using qPCR with primers specific to the regulated gene promoter. The ChIP primers used for Nox4 promoter detection were Nox4: ATGTCTGCAGCTGGACAGG and ACCGAAAGGAGCGATCAGT. All reactions were run in triplicate.

Cell-surface biotinylation assay. HEK293 cells were grown to $70 \%$ to $80 \%$ confluence in 6-well plates coated with poly-D-lysine (Sigma-Aldrich). Transient transfections with HA-p.Phe508del-CFTR and HA-WT-CFTR pCDNA3.1 plasmids were performed using Lipofectamine LTX Transfection Reagent (Invitrogen), according to the manufacturer's instructions. The day after transfection, cells were treated for 2, 6, or 24 hours with $10 \mu \mathrm{g} / \mathrm{mL}$ of anakinra. Cells were then placed at $4^{\circ} \mathrm{C}$ and washed 3 times with $\mathrm{PBS} / \mathrm{CaCl}_{2} / \mathrm{MgCl}_{2}$ (PBS $+2.5 \mathrm{mM} \mathrm{CaCl}$, $1 \mathrm{mM} \mathrm{MgCl} 2, \mathrm{pH}$ 7.4). Plasma membrane proteins were then biotinylated by gently shaking the cells in a $\mathrm{PBS} / \mathrm{CaCl}_{2} / \mathrm{MgCl}_{2}$ buffer containing EZ-Link Sulfo-NHS-SS-Biotin (Thermo Fisher Scientific) for 30 minutes. Some cells were permeabilized with $0.05 \%$ Triton X-100 in cold PBS/ $\mathrm{CaCl}_{2} / \mathrm{MgCl}_{2}$ for 10 minutes before biotin treatment. After biotinylation, cells were washed extensively with quenching buffer $(50 \mathrm{mM}$ glycine in $\mathrm{PBS} / \mathrm{CaCl}_{2} / \mathrm{MgCl}_{2}$ ) to remove excess biotin and then washed twice with PBS. The cells were then lysed and incubated overnight at $4^{\circ} \mathrm{C}$ with avidin solution (NeutrAvidin Plus UltraLink Resin, Pierce). Avidin-bound complexes were pelleted and washed 3 times by rotating the mixture for 5 minutes at $4^{\circ} \mathrm{C}$. Biotinylated proteins were eluted in a $1 \times$ sample buffer, resolved by SDS-PAGE, electrotransferred, and immunoblotted with the anti-CFTR antibody 596 CFTR (provided by JR Riordan through a program of the Cystic Fibrosis Foundation Therapeutics [CFFT], Charlotte, North Carolina, USA) and anti-calnexin (Abcam, ab22595).

Immunofluorescence and IHC. LC3 or AhR immunofluorescence were done on lung sections by staining with anti-LC3b (Abcam, ab48394) or anti-AhR (Proteintech, 17840-1-AP) antibody and secondary labeled antibodies. For CFTR immunofluorescence on CFBE410- cells, cells were treated with $10 \mu \mathrm{g} / \mathrm{mL}$ anakinra at $37^{\circ} \mathrm{C}$ for 4 hours, fixed in $2 \%$ formaldehyde for 15 minutes at room temperature, and permeabilized in blocking buffer containing 5\% FBS, 3\% BSA, and 0.5\% Triton X-100 in PBS. The cells were then incubated at $4^{\circ} \mathrm{C}$ with the primary antibody anti-CFTR (clone CF3, Abcam) and/or anti-GRASP55 (Proteintech, 10598-1-AP). After extensive washing with PBS, the slides were incubated at room temperature for 60 minutes with goat anti-mouse antibody to CFTR followed by Alexa Fluor 555 (BioLegend, 405324) and Alexa Fluor 488 anti-phalloidin (Thermo Fisher Scientific, A12379) for Factin labeling. Cell were pretreated with SiRNA for GRASP55 or $10 \mu \mathrm{g} /$ $\mathrm{mL}$ brefeldin A (Sigma-Aldrich) for 24 or 6 hours, respectively, at $37^{\circ} \mathrm{C}$ before immunostaining. For IHC, the tissues were removed and fixed in $10 \%$ phosphate-buffered formalin, embedded in paraffin, and sectioned at $5 \mu \mathrm{m}$. Sections were then rehydrated, and after antigen retrieval in citrate buffer (10 mM, pH 6.0), sections were fixed in $4 \%$ formaldehyde for 40 minutes at room temperature and permeabilized in blocking buffer containing 5\% FBS, 3\% BSA, and 0.5\% Triton X-100 in PBS. The sections were incubated at $4^{\circ} \mathrm{C}$ overnight with anti-CFTR (CF3, Abcam) antibody followed by biotinylated secondary antibodies. DAPI and hematoxylin were used to counterstain nuclei. All images were acquired 
using a BX51 fluorescence microscope (Olympus) with $\times 20$ and $\times 100$ objectives using analySIS image-processing software (Olympus).

Functional analysis of CFTR. Patch-clamp recordings were performed from p.Phe508del-CFTR-transfected FRT cells treated with $10 \mu \mathrm{g} / \mathrm{mL}$ anakinra, $3 \mu \mathrm{M}$ VX-809 (Aurogene), or vehicle for 4 hours at $37^{\circ} \mathrm{C}$. Pipettes with resistance of $2-4 \mathrm{M} \Omega$ were pulled from borosilicate glass capillary tubing (1B150F-3; World Precision Instruments), using a 5-step horizontal puller from Sutter Instrument. Cells were stimulated with forskolin $(10 \mu \mathrm{M})$, genistein $(30 \mu \mathrm{M})$, or $10 \mu \mathrm{M}$ VX-770 (Aurogene). Macroscopic CFTR currents were recorded in whole-cell dialyzed configuration using an EPC-10 patch-clamp amplifier (HEKA Elektronik). $\mathrm{I}-\mathrm{V}$ relationships were built by clamping the membrane potential of FRTcells at $-40 \mathrm{mV}$ and by delivering ramps from $-100 \mathrm{mV}$ to $50 \mathrm{mV}$. The pipette solution contained (mM): $113 \mathrm{~L}$-aspartic acid, $113 \mathrm{CsOH}, 27 \mathrm{CsCl}$, $1 \mathrm{NaCl}, 1 \mathrm{MgCl}_{2}, 1 \mathrm{EGTA}$, and $10 \mathrm{TES}$ (pH 7.2). $\operatorname{MgATP}(3 \mathrm{mM})$ was added just before patch-clamp experiments were started. The external solution contained the following (mM): $145 \mathrm{NaCl}, 4 \mathrm{CsCl}, 1 \mathrm{CaCl}_{2}, 10$ glucose, and 10 TES (pH 7.4). Results were analyzed with FITMASTER software (HEKA Elektronik) and Microcal Origin 8.0. A Kolmogorov-Smirnov normality test turned out to be nonsignificant. The variance was similar in the groups being compared. We considered all $P$ values of 0.05 or less to be significant. Ussing chamber experiments were performed as described (58). Chamber solution was buffered by bubbling with a mixture of $95 \% \mathrm{O}_{2}$ and $5 \% \mathrm{CO}_{2}$. $\mathrm{HBE}$ cells were short-circuited using $\mathrm{Ag} /$ $\mathrm{AgCl}$ agar electrodes. Short-circuit current and resistance were acquired or calculated using the VCC-600 transepithelial clamp from Physiologic Instruments and Acquire \& Analyze 2.3 software for data acquisition as previously described (58). A basolateral-to-apical chloride gradient was established by replacing $\mathrm{NaCl}$ with sodium gluconate in the apical (luminal) compartment to create a driving force for CFTR-dependent $\mathrm{Cl}^{-}$secretion. CFTR channels present at the apical surface of the epithelium (lumen side of the tissue) were activated. Stimulations with forskolin, CFTR inhibitor 172, and amiloride were performed as described (58).

Trp metabolites quantification by LC high-resolution mass spectrometry. The frozen cell samples $\left(3 \times 10^{6}\right)$ were thawed at $4^{\circ} \mathrm{C}$ and spiked with a suitable concentration of labeled internal standards (kynurenic acid-d5 and tryptophan-d5) in $2 \mathrm{~mL}$ tubes. After the addition of $250 \mu \mathrm{L}$ of a solution urea/Tris $\mathrm{HCl}(\mathrm{pH} 7.4)$, the tubes were vortexed for 15 seconds and sonicated for 15 minutes. Then, $750 \mu \mathrm{L}$ of acetonitrile was added and samples vortexed and centrifuged (16,800g, 15 minutes). The supernatants were transferred into a $2 \mathrm{~mL}$ tube. After evaporation under nitrogen stream $\left(40^{\circ} \mathrm{C}\right)$, the samples were resuspended in $100 \mu \mathrm{L}$ of a mixture $\mathrm{H}_{2} \mathrm{O} / \mathrm{MeOH}$ 95:5 (v/v), shaken, and then injected in the LC high-resolution mass spectrometer (LC-HRMS) system. Chromatographic separation was performed on the Ultimate 3000 HPLC system (Thermo Fisher Scientific). Stock solutions $(100 \mathrm{mg} / \mathrm{mL})$ were prepared by dissolving each standard in $\mathrm{H}_{2} \mathrm{O} / \mathrm{MeOH}, 90: 10$ (v/v), except kynurenic acid and kynurenic acid-d5, which were dissolved in DMSO. Analytes were separated on a XSelect HSS T3 column $(100 \times 2.1 \mathrm{~mm}, 1.7 \mu \mathrm{m}$, Waters Corporation) connected with a guard column $(2.1 \times 5 \mathrm{~mm}$, Waters Corporation). LC eluents A and B were water and acetonitrile (ACN), both containing $0.1 \%(\mathrm{v} / \mathrm{v})$ formic acid. From 0 to 9 minutes, the percentage of eluent $\mathrm{B}$ increased from $0 \%$ to $15 \%$ and from 9 to 9.9 minutes to $100 \%$. This condition was maintained for 1 minute and then the system returned to $0 \%$ B (0.1 minute) and it was reequilibrated for 2.5 minutes (run time: 13.5 minutes). The column and autosampler temperatures were kept at $40^{\circ} \mathrm{C}$ and $16^{\circ} \mathrm{C}$, respectively. The flow rate was $0.30 \mathrm{~mL} \mathrm{~min}{ }^{-1}$ and injection volume $10 \mu \mathrm{L}$. The mass spectrometer Q-Exactive Plus (Thermo Fisher Scientific) was equipped with a heated electrospray ionization (HESI-II) source. The HESI-II temperature was set at $350^{\circ} \mathrm{C}$, the capillary temperature at $300{ }^{\circ} \mathrm{C}$, and the electrospray voltage at $4.00 \mathrm{kV}$ (positive mode). Sheath and auxiliary gas were 40 and 15 arbitrary units. The mass analyzer was controlled by Xcalibur 3.0 software (Thermo Fisher Scientific). The acquisition was achieved in full scan/dd-MS ${ }^{2}$ mode. Quantitative analysis was performed using full scan data. The mass scan range was $\mathrm{m} / z 100$ to 500 . The data were acquired at a resolving power of 70000 full-width at half-maximum (FWHM) $(m / z 200)$. Automatic gain control (AGC) was set at $1 \times 10^{6}$ ions for a maximum injection time of 320 $\mathrm{ms}$. The precursor ions, filtered by the quadrupole (isolation window equal to $\mathrm{m} / \mathrm{z}$ 1.0), were fragmented with stepped normalized collision energies at 120, 30, and $50 \mathrm{eV}$. An inclusion list for $\mathrm{dd}-\mathrm{MS}^{2}$ experiments was used, including for each analyte the $m / z$ of precursor ion and expected retention time ( \pm 1 minute). A resolving power of $17,500 \mathrm{FWHM}(\mathrm{m} / \mathrm{z}$ 200) was used for dd-MS ${ }^{2}$ experiments with AGC target set at $5 \times 10^{5}$ ions and injection time at $80 \mathrm{~ms}$. Kynurenine and its metabolites were quantified by applying isotopic dilution methodology.

Senescence assay. MEF cells from passage 4 to 7 were seeded at $1.5 \times$ $10^{5}$ cells per well in 24 -well plates and exposed to $10 \mu \mathrm{g} / \mathrm{mL}$ anakinra at $37^{\circ} \mathrm{C}$. Triplicate dishes were counted at each passage. MEF cells at passage 6 were subjected to immunofluorescence staining by incubation, after fixation and permeabilization, with the rat monoclonal anti-p19Arf (Merck KGaA, Calbiochem, CB1012) as a marker for senescence, followed by the secondary antibody Alexa Fluor 555 Texas red-conjugated anti-rat IgG (Invitrogen, A-21434). DAPI was used to detect nuclei.

ELISA. Cytokine content was determined in lung homogenates by using specific ELISA kits according to the manufacturers' instructions (BioLegend, eBioscience Inc., and R\&D Systems). Kynurenine production was evaluated in cell supernatants (LDN). The cytokine and kynurenine concentrations were expressed as $\mathrm{pg} / \mathrm{mL}$ or $\mathrm{nmol} / \mathrm{L}$, respectively.

$R T$-PCR. RT-PCR was performed using the CFX96 Touch Real-Time PCR detection system and iTaq Universal SYBR Green Supermix (BioRad). Lung and cells were lysed and total RNA was isolated with TRIzol Reagent (Thermo Fisher Scientific), and cDNA was synthesized using the PrimeScript RT Reagent Kit with gDNA Eraser (Takara), according to the manufacturer's instructions. Each data point was examined for integrity by analysis of the amplification plot. The thermal profile for SYBR Green RT-PCR was at $95^{\circ} \mathrm{C}$ for 3 minutes followed by 40 cycles of denaturation for 30 seconds at $95^{\circ} \mathrm{C}$ and an annealing/extension step of 30 seconds at $60^{\circ} \mathrm{C}$. Amplification efficiencies were validated and normalized against Gapdh. The mouse primers $\left(5^{\prime}-3^{\prime}\right)$ were as follows: Gapdh: TCGTCCCGTAGACAAAATGG and TTGAGGTCARGAAGGGGTC;Ahrr:AGAGGGTTCCCCGTGCAG and ACTCACCACCAGAGCGAAGC; Cyp1 1 1: ACAGTGATTGGCAGAGATCG and GAAGGGGACGAAGGATGAAT Cyp1b1: TTCTCCAGCTTTTTGCCTGT and TAATGAAGCCGTCCTTGTCC; Gpx7: CCCATTCCTGAACCTTTCAA and GCACACGAAACCCCTGTACT; Ido1: CATGACATACGAGAACATGGAC and GACAGATATATGCGGAGAACG; Ido2: CATGGCGCTGGCCGCTATCA and TTAAGGCCGGGCACTGCTGC; Nox4: GAAGATTTGCCTGGAAGAACC and AGGTTTGTTGCTCCTGATGC; $p 22^{\text {phox }}$ : ACCTGACCGCTGTGGTGAA and GTGGagGaCAGCCCGGA; Sod3: CCTAGCAGACAGGCTTGACC and GTCGTCCTAGCTCCATCCAG; Tdo2: ATGAGTGGGTGCCCGTTTG and GGCTCTGTTTACACCAGTTTGAG.

QuantiGene Plex gene expression assay. Total RNA from experiment was extracted with TRIzol reagent (Thermo Fisher Scientific) according to the 
manufacturer's instructions. The total RNA was qualified and quantified by the Tecan Infinite 200 microplate reader (Tecan Group Ltd) following the instrument's protocols. The RNA was diluted using nuclease-free water. The sample input range was $250 \mathrm{ng} /$ well. All QuantiGene probe sets are available from Thermo Fisher Scientific, and the individual accession numbers and probe set regions are summarized in Supplemental Table 4. The QuantiGene assays were performed as indicated in the manufacturer's protocol. Plates were read using Magpix (Luminex). Data analysis was performed by normalizing the signals obtained for the genes of interest to the geometric mean of the reference gene signals (Polr2a, Tfrc, and Hprt).

Statistics. GraphPad Prism software 6.01 was used for the analysis. Statistical significance was calculated by 1 or 2-way ANOVA (Tukey's or Bonferroni's post hoc test) for multiple comparisons and by a 2-tailed Student's $t$ test for single comparisons. The distribution of levels tested by Kolmogorov-Smirnov normality test turned out to be nonsignificant. The variance was similar in the groups being compared. We considered all $P$ values of 0.05 or less to be significant.

The colocalization program ImageJ (59) with the JACoP plug-in was used to quantify the degree of overlap by calculating the colocalization coefficients (Pearson's correlation coefficient, overlap coefficient according to Manders, and the overlap coefficients as reported in Supplemental Figure 8).

Study approval. Mouse experiments were performed according to Italian Approved Animal Welfare authorization 360/2015-PR and Legislative Decree 26/2014 regarding the animal license approved by the Italian Ministry of Health (Rome) lasting for 5 years (2015-2020).

\section{Author contributions}

FLVDV and LR conceived and designed experiments. ADL, M. Pariano, RGI, MB, and GR performed in vivo experiments. M. Pariano, VO, FDO, CS, M. Puccetti, MMB, SG, MC, and MADF contributed to Western blot and IP, ELISA, RT-PCR, immunofluorescence, and IHC experiments. VRV and LM performed Ussing chamber experiments. DP and GS analyzed RNA-Seq data. SP performed ChIP experiments. BC performed limited proteolysis experiments. FF, MG, and GM performed the luciferase reporter experiments; RG and CB performed LC-HRMS; LS performed patch clamp experiments. LR wrote the paper. FLVDV, VNT, CC, and CAD edited the paper. LR supervised the work.

\section{Acknowledgments}

This study was supported by grants funded by the Italian Cystic Fibrosis Research Foundation (research projects FFC22/2014 and FFC17/2020 to SG and LR). FLVDV was supported by a VIDI grant from NWO ZonMw, Netherlands. CAD is supported by NIH grant AI 15614. M. Pariano, CS, GR, and M. Puccetti gratefully acknowledge a fellowship from the Italian Cystic Fibrosis Research Foundation. We thank the primary cell culture service offered from the Italian Cystic Fibrosis Research Foundation for kindly providing us with the HBE cells. We thank B. Scholte, Erasmus Medical Center Rotterdam, Netherlands, who provided CftrmIEUR mice (F508del mice, European Economic Community European Coordination Action for Research in Cystic Fibrosis program EU FP6 SHMCT-2005-018932). We thank Cristina Massi-Benedetti for superb editorial assistance.

Address correspondence to: Frank L. van de Veerdonk, Department of Medicine, Radboudumc Center for Infectious Diseases (RCI), Nijmegen, Netherlands. Phone: 0031.641100754; Email: frank.vandeveerdonk@radboudumc.nl. Or to: Luigina Romani, Department of Medicine and Surgery, University of Perugia, Polo Unico Sant'Andrea delle Fratte, 06132 Perugia, Italy. Phone: 39.075.5858234; Email: luigina.romani@unipg.it.
1. Bouchecareilh M, Balch WE. Proteostasis: a new therapeutic paradigm for pulmonary disease. Proc Am Thorac Soc. 2011;8(2):189-195.

2. Mizushima N. A brief history of autophagy from cell biology to physiology and disease. Nat Cell Biol. 2018;20(5):521-527.

3. Cadwell K. Crosstalk between autophagy and inflammatory signalling pathways: balancing defence and homeostasis. Nat Rev Immunol. 2016;16(11):661-675.

4. Balch WE, et al. Malfolded protein structure and proteostasis in lung diseases. Am J Respir Crit Care Med. 2014;189(1):96-103.

5. Elborn JS. Cystic fibrosis. Lancet. 2016;388(10059):2519-2531.

6. Bodas M, Vij N. Adapting proteostasis and autophagy for controlling the pathogenesis of cystic fibrosis lung disease. Front Pharmacol. 2019;10:20.

7. Maiuri L, et al. Strategies for the etiological therapy of cystic fibrosis. Cell Death Differ. 2017;24(11):1825-1844.

8. Luciani A, et al. Defective CFTR induces aggresome formation and lung inflammation in cystic fibrosis through ROS-mediated autophagy inhibition. Nat Cell Biol. 2010;12(9):863-875.

9. Luciani A, et al. Targeting autophagy as a novel strategy for facilitating the therapeutic action of potentiators on $\Delta \mathrm{F} 508$ cystic fibrosis transmembrane conductance regulator. Autophagy.
2012;8(11):1657-1672.

10. Cavalli G, Dinarello CA. Anakinra therapy for non-cancer inflammatory diseases. Front Pharmacol. 2018;9:1157.

11. Dinarello CA, et al. Treating inflammation by blocking interleukin-1 in a broad spectrum of diseases. Nat Rev Drug Discov. 2012;11(8):633-652.

12. Gabay C, et al. Mouse IL-1 receptor antagonist isoforms: complementary DNA cloning and protein expression of intracellular isoform and tissue distribution of secreted and intracellular IL-1 receptor antagonist in vivo. J Immunol. 1997;159(12):5905-5913.

13. Banda NK, et al. Intracellular IL-1 receptor antagonist type 1 inhibits IL-1-induced cytokine production in keratinocytes through binding to the third component of the COP9 signalosome. Jimmunol. 2005;174(6):3608-3616.

14. Iannitti RG, et al. IL-1 receptor antagonist ameliorates inflammasome-dependent inflammation in murine and human cystic fibrosis. Nat Commun. 2016;7:10791.

15. Vecile E, et al. Intracellular function of interleukin-1 receptor antagonist in ischemic cardiomyocytes. PLoS One. 2013;8(1):e53265.

16. Dripps DJ, et al. Interleukin-1 (IL-1) receptor antagonist binds to the $80-\mathrm{kDa}$ IL-1 receptor but does not initiate IL-1 signal transduction. J Biol Chem. 1991;266(16):10331-10336.
17. de Luca A, et al. IL-1 receptor blockade restores autophagy and reduces inflammation in chronic granulomatous disease in mice and in humans. Proc Natl Acad Sci US A. 2014;111(9):3526-2531.

18. Armstrong H, et al. Nigericin promotes NLRP3independent bacterial killing in macrophages. Front Immunol. 2019;10:2296.

19. Evans RJ, et al. Mapping receptor binding sites in interleukin (IL)-1 receptor antagonist and IL-1 beta by site-directed mutagenesis. Identification of a single site in IL-1ra and two sites in IL-1 beta. J Biol Chem.1995;270(19):11477-11483.

20. Filomeni $G$, et al. Oxidative stress and autophagy: the clash between damage and metabolic needs. Cell Death Differ. 2015;22(3):377-388.

21. Forte M, et al. Functional role of Nox 4 in autophagy. Adv Exp Med Biol. 2017;982:307-326.

22. Takac I, et al. The E-loop is involved in hydrogen peroxide formation by the NADPH oxidase Nox4. JBiol Chem. 2011;286(15):13304-13313.

23. Graham KA, et al. NADPH oxidase 4 is an oncoprotein localized to mitochondria. Cancer Biol Ther. 2010;10(3):223-231.

24. Scherz-Shouval R, et al. Reactive oxygen species are essential for autophagy and specifically regulate the activity of Atg4. EMBO J. 2007;26(7):1749-1760.

25 . Tai H, et al. Autophagy impairment with lysosomal and mitochondrial dysfunction is an important characteristic of oxidative stress-induced senescence. 
Autophagy. 2017;13(1):99-113.

26. Klotz LO, Steinbrenner H. Cellular adaptation to xenobiotics: Interplay between xenosensors, reactive oxygen species and FOXO transcription factors. Redox Biol. 2017;13:646-654.

27. Huai W, et al. Aryl hydrocarbon receptor negatively regulates NLRP 3 inflammasome activity by inhibiting NLRP3 transcription. Nat Commun. 2014;5:4738.

28. Tsai $\mathrm{CH}$, et al. The inhibition of lung cancer cell migration by AhR-regulated autophagy. Sci Rep. 2017;7:41927.

29. McGuire J, et al. A cellular factor stimulates ligand-dependent release of hsp 90 from the basic helix-loop-helix dioxin receptor. Mol Cell Biol. 1994;14(4):2438-2446.

30. Orabona C, et al. SOCS3 drives proteasomal degradation of indoleamine 2,3-dioxygenase (IDO) and antagonizes IDO-dependent tolerogenesis. Proc Natl Acad Sci U S A. 2008;105(52):20828-20833.

31. Bessede A, et al. Aryl hydrocarbon receptor control of a disease tolerance defence pathway. Nature. 2014;511(7508):184-190.

32. Mohammadi-Bardbori A, et al. NADPH oxidasedependent mechanism explains how arsenic and other oxidants can activate aryl hydrocarbon receptor signaling. Chem Res Toxicol. 2015;28(12):2278-2286.

33. Galluzzi L, et al. Molecular definitions of autophagy and related processes. EMBOJ. 2017;36(13):1811-1836.

34. Urano $Y$, et al. 6-Hydroxydopamine induces secretion of PARK7/DJ-1 via autophagy-based unconventional secretory pathway. Autophagy. 2018;14(11):1943-1958.

35. Gee HY, et al. Rescue of $\triangle$ F508-CFTR trafficking via a GRASP-dependent unconventional secretion pathway. Cell. 2011;146(5):746-760.

36. Quartier P, et al. A multicentre, randomised, double-blind, placebo-controlled trial with the interleukin-1 receptor antagonist anakinra in patients with systemic-onset juvenile idiopathic arthritis (ANAJIS trial). Ann Rheum Dis. 2011;70(5):747-754.

37. Nguyen NT, et al. Aryl hydrocarbon receptor negatively regulates dendritic cell immunogenicity via a kynurenine-dependent mechanism. Proc Natl Acad SciUSA. 2010;107(46):19961-19966.

38. Block K, et al. Subcellular localization of Nox 4 and regulation in diabetes. Proc Natl Acad Sci U S A. 2009;106(34):14385-14390.

39. Pariano M, et al. Anakinra activates superoxide dismutase 2 to mitigate inflammasome activity. Int JMol Sci. 2021;22(12):6531.

40. Green DR, et al. Mitochondria and the autophagy-inflammation-cell death axis in organismal aging. Science. 2011;333(6046):1109-1112.

41. Fritzsching B, et al. Hypoxic epithelial necrosis triggers neutrophilic inflammation via IL-1 receptor signaling in cystic fibrosis lung disease. Am J Respir Crit Care Med. 2015;191(8):902-913.

42. Chen G, et al. IL-1 $\beta$ dominates the promucin secretory cytokine profile in cystic fibrosis. J Clin Invest. 2019;129(10):4433-4450.

43. Zhou W, et al. Longitudinal multi-omics of host-microbe dynamics in prediabetes. Nature. 2019;569(7758):663-671.

44. Kyriazopoulou E, et al. Effect of anakinra on mortality in patients with COVID-19: a systematic review and patient-level meta-analysis. Lancet Rheumatol.2021;3(10):a systematic review and patient-e697.

45. Kyriazopoulou E, et al. Early treatment of COVID19 with anakinra guided by soluble urokinase plasminogen receptor plasma levels: a double-blind, randomized controlled phase 3 trial. Nat Med. 2021;27(10):1752-1760.

46. van Doorninck JH, et al. A mouse model for the cystic fibrosis delta F508 mutation. EMBOJ. 1995;14(18):4403-4411.

47. Castelli M, et al. Hepatocyte odd protein shuttling (HOPS) is a bridging protein in the nucleophosmin-p19 Arf network. Oncogene.
2013;32(28):3350-3358

48. Bruscia $\mathrm{E}$, et al. Isolation of $\mathrm{CF}$ cell lines corrected at DeltaF508-CFTR locus by SFHR-mediated targeting. Gene Ther. 2002;9(11):683-685.

49. Han D, et al. Comparison of recombinant cell bioassays for the detection of Ah receptor agonists. Biofactors. 2004;20(1):11-22.

50. Latypov RF, et al. Denaturant-dependent conformational changes in a beta-trefoil protein: global and residue-specific aspects of an equilibrium denaturation process. Biochemistry. 2009;48(46):10934-10947.

51. Conesa A, et al. Blast2GO: a universal tool for annotation, visualization and analysis in functional genomics research. Bioinformatics. 2005;21(18):3674-3676.

52. Ye J, et al. WEGO: a web tool for plotting GO annotations. Nucleic Acids Res. 2006;34(w1):W293-297.

53. Klionsky DJ, et al. Guidelines for the use and interpretation of assays for monitoring autophagy (3rd edition). Autophagy. 2016;12(1):1-222.

54. Iannitti RG, et al. Hypoxia promotes danger-mediated inflammation via receptor for advanced glycation end products in cystic fibrosis. Am J Respir Crit Care Med. 2013;188(11):1338-1350.

55. Peshavariya HM, et al. Analysis of dihydroethidium fluorescence for the detection of intracellular and extracellular superoxide produced by NADPH oxidase. Free Radic Res. 2007;41(6):699-712.

56. Bylund J, et al. Intracellular generation of superoxide by the phagocyte NADPH oxidase: how, where, and what for? Free Radic Biol Med. 2010;49(12):1834-1845.

57. Cathcart R, et al. Detection of picomole levels of hydroperoxides using a fluorescent dichlorofluorescein assay. Anal Biochem. 1983;134(1):111-116.

58. Romani L, et al. Thymosin alpha1 represents a potential potent single-molecule-based therapy for cystic fibrosis. Nat Med. 2017;23(5):590-600.

59. Collins TJ. Image for microscopy. Biotechniques. 2007;43(1 suppl):25-30. 